\title{
Epidemiological Mucormycosis treatment and diagnosis challenges using the adaptive properties of computer vision techniques based approach: a review
}

\author{
Nira ${ }^{1} \cdot$ Harekrishna Kumar ${ }^{1}$
}

Received: 12 June 2021 / Revised: 13 September 2021 / Accepted: 25 January 2022 /

Published online: 25 February 2022

(C) The Author(s), under exclusive licence to Springer Science+Business Media, LLC, part of Springer Nature 2022

\begin{abstract}
As everyone knows that in today's time Artificial Intelligence, Machine Learning and Deep Learning are being used extensively and generally researchers are thinking of using them everywhere. At the same time, we are also seeing that the second wave of corona has wreaked havoc in India. More than 4 lakh cases are coming in $24 \mathrm{~h}$. In the meantime, news came that a new deadly fungus has come, which doctors have named Mucormycosis (Black fungus). This fungus also spread rapidly in many states, due to which states have declared this disease as an epidemic. It has become very important to find a cure for this life-threatening fungus by taking the help of our today's devices and technology such as artificial intelligence, data learning. It was found that the CT-Scan has much more adequate information and delivers greater evaluation validity than the chest $\mathrm{X}$-Ray. After that the steps of Image processing such as pre-processing, segmentation, all these were surveyed in which it was found that accuracy score for the deep features retrieved from the ResNet50 model and SVM classifier using the Linear kernel function was $94.7 \%$, which was the highest of all the findings. Also studied about Deep Belief Network (DBN) that how easy it can be to diagnose a life-threatening infection like fungus. Then a survey explained how computer vision helped in the corona era, in the same way it would help in epidemics like Mucormycosis.
\end{abstract}

Keywords Mucormycosis $\cdot$ Computer vision · Black fungus $\cdot$ Artificial intelligence $\cdot$

Deep learning

Harekrishna Kumar

harekrishnabgp@gmail.com

1 Department of Electronics and Communication, GLA University, Mathura 281406, India 


\section{Introduction}

At present, the country is facing the second wave of Corona epidemic. Every day the figures of the number of infections and deaths are released, they scare $[4,8]$. But in the midst of all this, problems are also being faced by those people who are facing various difficulties after post Covid. People recovering from corona infection are facing problems like swelling in the eyes, itching, hearing problems, headache. Black fungal infection (Mucormycosis) is being said to be responsible for all this. In fact, there are tiny fungi in the air called Mucormycitis. The name of this disease is Mucormycosis. In Fig. 1 has shown the image of Mucormycosis. It was earlier called Zygomycosis. It is a type of fungal infection. Usually this infection usually starts in the nose. Which gradually spreads to the eyes. Treatment is necessary as soon as its infection spreads [12, 27, 29]. It can paralyze the area around the iris. If the infection spreads for a long time, the risk of loss of eyesight increases. Mucormycosis can be more dangerous for corona patients with diabetes. It is very important for corona patients with diabetes to avoid it. If it is treated at the right time, then the problem can be reduced. This is not because the infection is of black color, but because it is named because after this the eyesight goes away. If the infection reaches a more severe stage, the risk of meningitis and sinus clotting also increases. Earlier 12 cases were reported in Sir Gangram Hospital in Delhi. These corona patients have had Mucormycosis infection. If it is not treated within 15 days, it can infect the brain as well. In such a situation, the most important thing is not to be afraid in any way, but to be alert so that treatment can be received at the right time [13].

According to the news published in the Indian Express newspaper, Dr. Manish Munjal, Senior ENT Surgeon of Sir Ganga Ram Hospital said that cases of Mucormycosis have been seen in serious corona patients [6]. We are on full alert. If there is swelling in the nose, eyes or throat of a corona patient, then we immediately examine him to see if he has a black fungus. Antifungal therapy

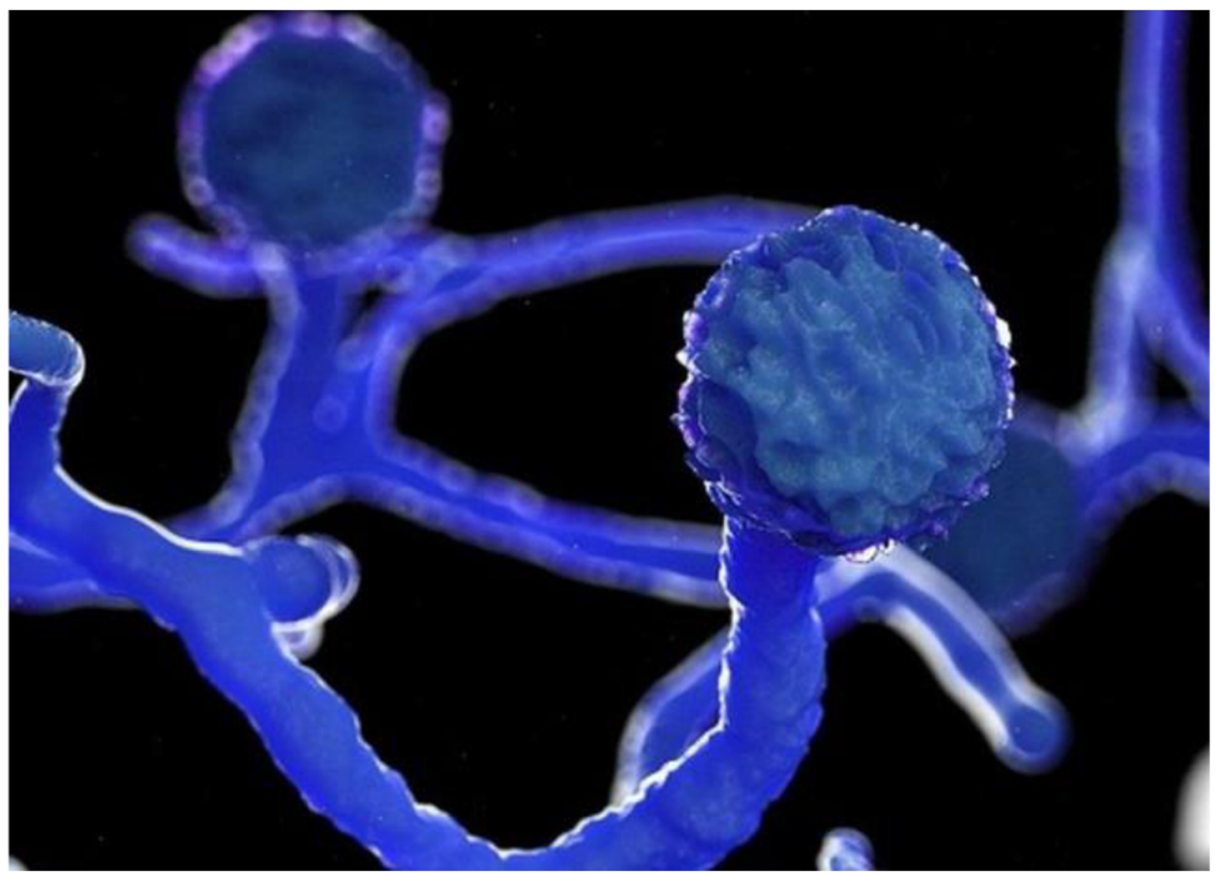

Fig. 1 Mucormycosis: the 'black fungus' [5] 
is started by taking immediate biopsy of such patients so that the infection does not spread to the eyes. Mucormycosis infection is also engulfing the post covid patient, patients who have been cured of corona. That's why all the doctors of the country have been asked to be alert about post covid patients. Take the side effects that occur in them seriously, so that they do not cause any kind of problem and can be treated on time. When a patient who has recovered from corona breathes, these fungi settle in the lungs along with his sinus cavity [7, 12]. However, are these fungi or steroids responsible for the kind of problems that post covid patients are facing. There is no definite information available in this regard yet. But it is clear from the kind of trouble most people are describing that they are getting two or four from this problem in some form or the other.

\section{Black fungus infection in India: Mucormycosis spreading in India}

Black fungus is a very dangerous infection, which till now used to be very few people, but in the last few days it has become a victim of Covid-19 patients. Black fungus epidemic is taking a formidable form these days. Thousands of people have been affected by Mucormycosis after recovering from Covid. In India, rising cases of Black Fungus have raised concerns amid the second wave of Covid19. Due to this, many people have died in many parts of the country. The second wave of corona virus has already made people's lives difficult and in the meantime, the cases of black fungus are increasing continuously [12]. In such a situation, the central government has urged the states to declare it an epidemic under the Epidemic Act 1897. Due to the rapid spread of infection, it has now also been declared an epidemic. More than 12 thousand people in the country have come under the grip of black fungus after Covid and hundreds have died. More than 700 cases have been registered in Delhi alone and in Maharashtra alone, about 90 people have died due to this. In Fig. 2 has shown black spot of black fungus.

Apart from the capital, the number of people suffering from Mucormycosis in other states is increasing day by day. Black fungus is such a dangerous infection, which has been happening to very few people till now. One in a million had this infection. But during the last few days, this infection has spread very fast among patients infected with corona. Many a times, we ignore the

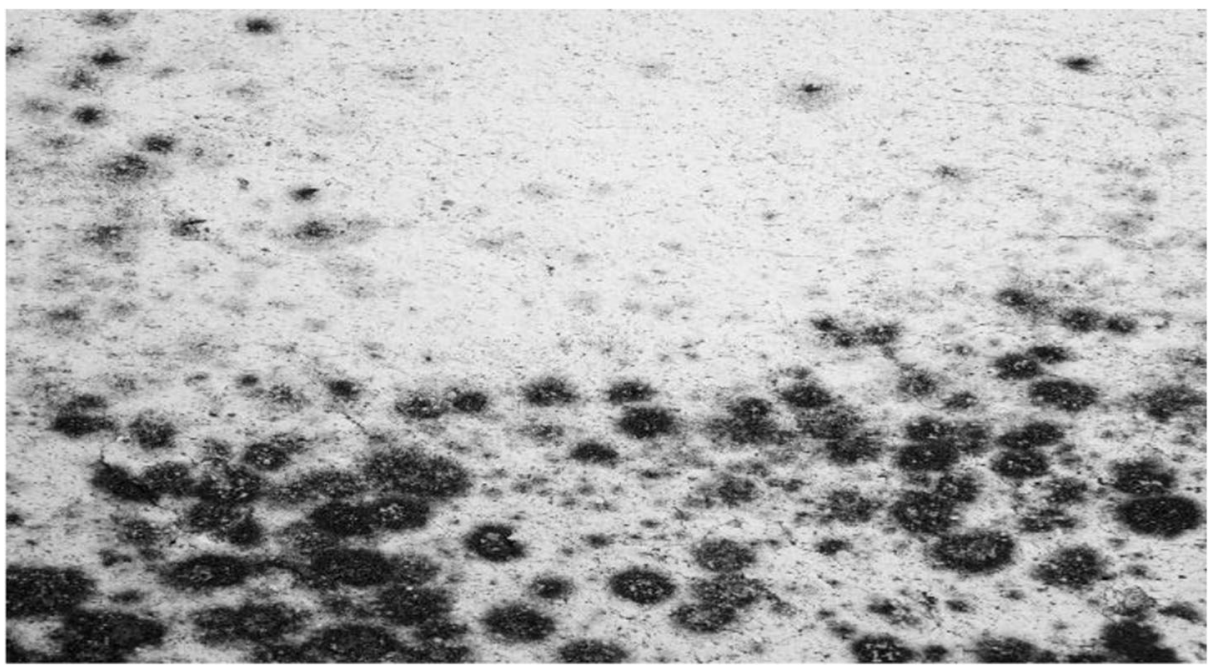

Fig. 2 Black spots of black fungus [7] 
common symptoms, which later become the cause of some serious disease. If you pay attention to these symptoms immediately and get treatment in time, then fungus can be avoided. Now there is also a shortage of medicines to remove this fungus[20,53]. A report by the US-based Centres for Disease Control and Prevention (CDC) has shown that the mortality rate of this deadly infection is estimated at 54\%. That is, it is as dangerous as cancer. As of June 7, 2021, the nation had 28,252 Mucormycosis confirmed cases from 28 states. There are 24,370 patients with a COVID-19 record and 17,601 instances with a diabetic record [66,67]. India had the greatest number of Mucormycosis cases (6329) [5]. The number of occurrences of Mucormycosis involving COVID-19 people is frightening, according to doctors. The majority of those people had diabetes and were being handled with steroids for SARS-CoV-2 disease, which could have caused them more vulnerable to fungal infection. Mucormycosis seems to be more prevalent among people who have a low immune system or who have had a bone marrow transplant with fewer neutrophils [35] [85]. COVID-19's introduction has resulted in a slew of new illnesses and problems [16] [63]. During India's second wave, there has been an increase in such cases [77]. Patients with a past of diabetes mellitus, as well as $95 \%$ of those with serious or critical COVID-19, have been found to have Mucormycosis caused by COVID-19 [11]. Furthermore, it has been noted that patients with really no previous record of any underlying illness might be confirmed with Mucormycosis after exposure to the fungus [65]. The incidence of Mucormycosis after COVID-19 infection can be explained in a number of ways. COVID-19 patients have a wide range of pulmonary alterations [91], which could be a point of entry for fungi. COVID-19 is also linked to immunological failure, as it prevents polymorphonuclear phagocytes from attacking fungal spores after they enter the body.

According to the central government data, currently the mortality rate due to black fungus in the country is around 3\% (3.02) but now it has registered an increase of $66 \%$ in its cases. Let us tell you that in India, most of those people who are recovering from Covid or those who are sugar patients are falling prey to black fungus [19]. Doctors believe that people are ignoring the symptoms of black fungus. Dr. NC Panwar, Senior Consultant of CL Panwar Hospital, Jaipur and member of the Homeopathic Medical Association of India, said that if the symptoms of black fungus are identified at the right time, then it can be avoided. The doctor said that these days those people who are recovering from Covid and who are diabetic patients need to be more cautious. Because $99 \%$ of these people are in the grip of black fungus. Speaking to Financial Express Online, Dr. Uday Phadke, Director (Department of Endocrinology and Diabetes) Pune-based Sahyadri Hospitals and Endocrinologist-Diabetologist said that in the first year there were one or two cases of black fungus, but now in Sahyadri Hospitals in Pune only. 30-35 cases are coming in a month. That is, one case every day. Actually, Black fungus infection occurs only to those with weak immunity $[72,74]$. Now since many people have become weak due to the attack of corona, then this fungal infection also increased. Whereas earlier this disease used to affect more on chemotherapy, uncontrolled sugar, people undergoing any kind of transplant and the elderly. This fungus reaches the rest of the body through the nose $[30,31]$. Usually this fungus is in the air and goes into the nose through the breath. Many times infection occurs even when the cut or burnt places of the body come in contact with this fungus. That is, the nose is the main place of its entry, but it can attack any part of the body. It is such a dangerous infection that about half of the victims lose their lives. There have also been some cases in which patients have had to remove their eyes to save them. Most cases of black fungus are coming in diabetic people. In such a situation, they need to be most careful and keep checking their sugar level regularly [80-82]. Dr. Shweta Budyal, Senior Consultant Endocrinologist at Fortis Hospital, Mulund, told The Indian Express that black fungus usually affects the sinuses, brain and lungs. Although according to Budyal, the oral 
cavity or brain is most likely to be affected by black fungus, but in many cases it can also affect other parts of the body such as the gastrointestinal tract, skin and other organ systems of the body. According to Dr. Budyal, if there are any of these symptoms, then black fungus should be investigated [31].

\subsection{Symptoms of Black fungus (deadly fungus)}

According to [38] paper, there is evidence that these are the main symptoms of black fungus.

i. Eye irritation- The most pain in this is in the eyes. From the nose and mouth to the attack, the black fungus reaches the eyes very quickly. If infected with this, re-transplant B of the eye is not possible [30].

ii. Face numbness- Black fungus also attacks the mouth and jaw through the nose. It has been seen in many patients that their face becomes numb. Numbness in hands and feet is common but face is rare. So if you feel like this too, then you need to get tested [30].

iii. Severe headache- According to Dr. NC Panwar, like Covid, black fungus also enters the body through the mouth and nose, which can cause severe headache. If you have recovered from Covid, slept properly and you're eating habits are also right, despite this you are feeling headache without any reason, then you should consult a doctor immediately. It is possible that a virus of black fungus has entered inside you [38].

iv. Dark spots around nose- Since black fungus enters through the nose, you should be alert if you see black spots around the nose. Such marks may be the initial symptoms of Mucormycosis. Normally the nose gets blocked when you get cold, cough and cold [40]. But after the Covid recovery, everything is fine for you, yet the nose suddenly gets blocked, then understand that the black fungus has caught you. Contact a doctor immediately in case of nasal congestion.

v. One side swollen Face-Experts say that due to black fungus, swelling can be seen in one part of your face. Even when your eyes fall on this area, there is a sharp pain. By the way, there are many reasons for swelling on the face, such as not sleeping properly at night or sleeping for a long time, due to the problem related to gums, swelling on the face also occurs [45].

vi. Tooth looseness and swollen gums- Unexplained pain in the teeth, swollen gums can also be a symptom of black fungus. If you are having any kind of looseness in the teeth or any kind of problem in the gums for a few days, then consult a doctor [30].

\subsection{Reasons behind increasing the no. of cases for Black fungus}

According to Dr. Shweta, steroids are being used in the treatment of corona, due to which the level of sugar is increasing. The risk of black fungus infection increases due to increased sugar level and lack of physical activity. The use of steroids lowers the body's immunity, due to which the body's immune system is not able to work effectively against black fungus [5]. According to Dr. V Mohan, Chief Consultant and Chairman of the Chennai-based Diabetes Specialties Centre, apart from diabetes, cases of black fungus are also increasing due to hygiene and contaminated equipment. Dr. Mohan says that corona cases are coming so much that not much attention is being paid to cleanliness in hospitals. Due to this, the possibility of fungus accumulation on the equipment increases [6]. 


\subsection{Caution is necessary in the treatment of Black fungus}

According to Dr. Shweta, it is very important to detect black fungus in diabetic patients at an early stage. He says that giving more strong medicines to diabetic people also puts them at risk of having a bad effect on their kidneys or other organs. According to Dr. Shweta, the corona infected who are getting black fungus, steroid dose should be given very carefully at the time of treatment and after recovery [12]. According to Dr. Phadke, the treatment of black fungus should be done in three stages. In the first phase, only the cause of infection should be detected and removed and sugar level and acidosis should be checked. After this, the dead tissue should be removed aggressively through surgery, so that the fungus can be prevented from spreading further. After this appropriate medicines should be given.

Corona virus infection is worldwide but cases of black fungus are coming only in India. According to experts, carelessness regarding diabetes in India, wearing the same mask over and over and the environmental conditions here can also be a reason. Along with the corona epidemic in the country, black fungus (Mucormycosis) is also wreaking havoc. Weak immunity and steroids are being blamed for this. Different theories are being presented by doctors on this. But the question is arising that the way black fungus is becoming uncontrollable in India, it is not being seen in any other country [31]. So far, more than 12 thousand black fungus cases have been reported across the country. Many states have already declared Mucormycosis as a notified disease under the Epidemic Act. Most of the victims of black fungus in India are patients of corona infection or sugar. According to doctors, there is an increased risk of diseases other than corona virus infection in patients with weak immunity in India. Fungal infections are believed to be flourishing, especially due to frequent use of dirty masks, high diabetes and in some cases industrial oxygen, on which people are more dependent, among other reasons. Apart from this, due to low immunity in the body, black and white fungal infections are also arising in patients. According to a doctor at Sharp Sight Eye Hospitals, Mucormycosis, or black fungus, has a 54\% mortality rate, according to data from the United States Centres for Disease Control and Prevention. Dr. B Kamal Kapoor, Director and Co-Founder, Sharp Sight Eye Hospitals, said that India's adult population has an estimated 73 million cases of diabetes [84, 86]. Using steroids to control the disease process also increases the level of diabetes leading to diabetic complications. In Indians, taking medicines on their own without doctor's consultation is also the reason for increasing diseases, due to which the recovery of patients takes longer than usual. Due to this, more complications are being created in the patients and many types of infections are also increasing. On this issue, Dr. Amit Goyal, ENT Head and Professor, Jodhpur AIIMS Hospital, told IANS that two things are important in India, many people do not check sugar daily or do not eat medicines. People believe that once you start medicine, you will have to take medicine for the rest of your life. Dr. Goyal said, 'I think there is no unmonitored steroid use in other countries than India. At present, when research is done on this, then it will be fully known why this happened? He further told that our lack of cleanliness here can also be a reason. People are reusing used masks. Is this also due to the large population of India? In response to this question, Dr. Goyal said that if we compare $1 \%$ population of US and India, then there will be a difference between the two because they are $1 \%$ in saying but the numbers will be different [87].

This can also be a reason, but the way cases are coming to us here, they are not visible in other places. The answer can be found only when the patients of diabetes of other countries are 
compared with their own country and it is seen that are there more prevalence of fungus in our country compared to the prevalence of diabetes in other countries. In Fig. 3 reported the states where humans are infected by black fungus. According to doctors, the specialty of black fungus is that the patient suffering from it can never sit at home, he has to go to the hospital. Corona-infected, people with low immune system who have been in ICU for a long time, cancer, chemotherapy patients, patients using steroids and patients suffering from uncontrolled diabetes are mostly getting infected with the fungus. Dr. (Prof.) Anil Arora, Chairman, Institute of Liver, Gastroenterology and Pancreaticobiliary Sciences, Sir Ganga Ram Hospital told IANS, "Most of the fungal infections in the world are reported from India. Other smaller countries have smaller populations and lower total cases. In India, even in the last phase of the second wave, 2 lakh cases of corona infection are coming. A total of 30 thousand corona infected patients have been reported in Australia". Apart from this, maximum cases of black fungus have been reported in India [38]. According to doctors, black fungus majorly affects the nostrils, sinuses, retinal vessels and brain in different ways. Dr. Ritu Saxena, head of the emergency department at Delhi's LNJP Hospital, told IANS that while we are taking steroids in excess, the environmental conditions here can also be a reason. The third reason is the use of industrial oxygen, excessive use of zinc. All these can also be the reason but they are only theory so far, nothing has been proved yet. In India, people were negligent, taking steroids even at home in case of medicines. Black fungus is being seen more in those patients who have taken care of their home or those who have been treated in private hospitals. Few such patients have been seen in government hospitals [95, 96].

Out of all the patients who have gone from LNJP Hospital, only a few patients came back for treatment, otherwise all the patients are from outside. Top 11 states reported the number of cases and deaths in Fig. 4. Although according to the information, doctors use an injection named Liposomal Amphoteresirin B to deal with this disease, to promote the production of this drug, the Government of India has given license to five more companies to make it [42].

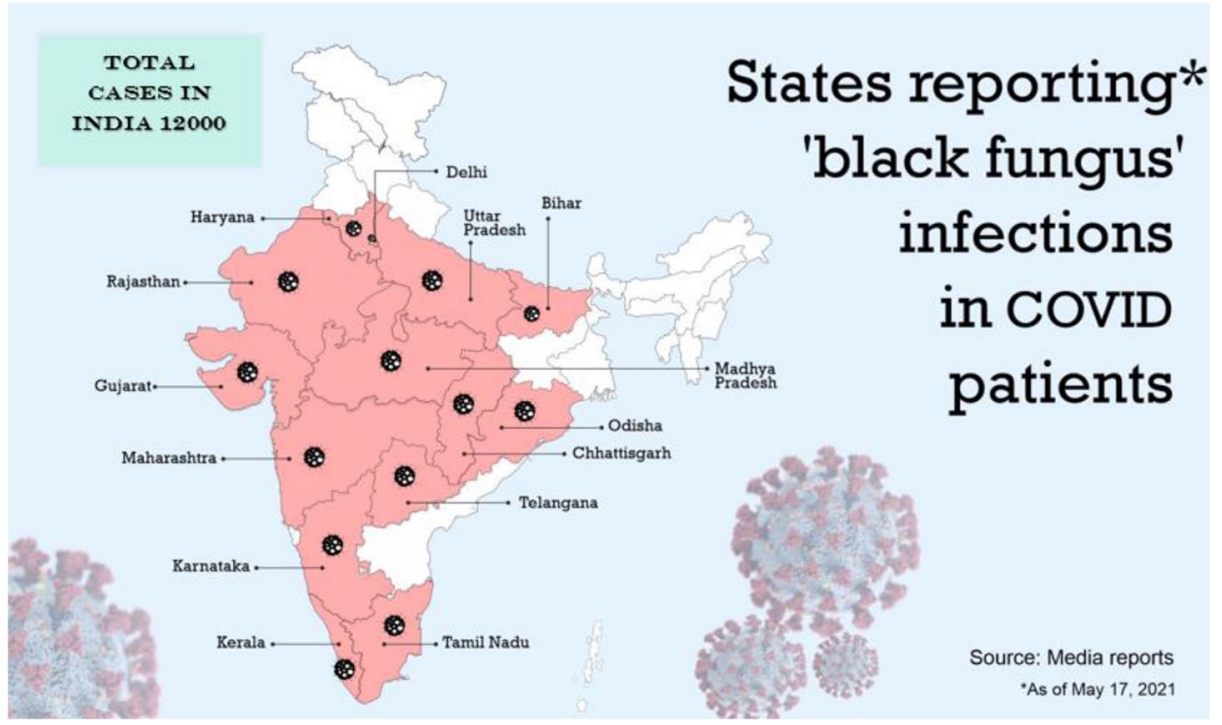

Fig. 3 States reporting black fungus infections in COVID patients [7] 


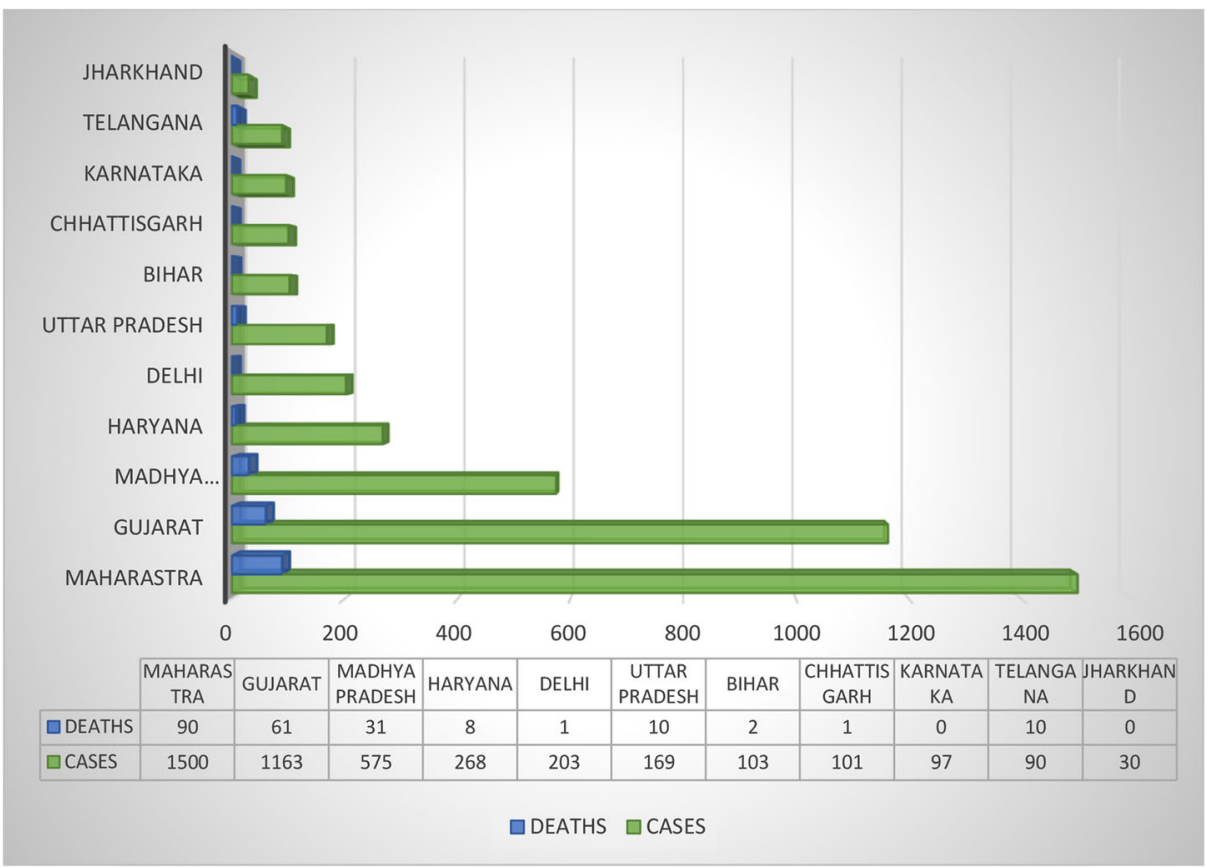

Fig. 4 Top 11 States information: number of cases and deaths as per state [32]

\section{White fungus: Which causes multiple perforations "throughout the intestine"}

\subsection{White fungus, which is considered more dangerous than black fungus}

Fungal infection continues to wreak havoc amid the corona epidemic. After the arrival of many patients of black fungus one after the other in many states, the central government asked the affected states to declare it an epidemic. The elimination of this infection could not even begin, that suddenly the patients of White Fungus also started coming. According to experts, this new infection is more dangerous than black fungus because it affects not only one organ, but every organ from lungs and brain. Days after cases of some white fungus found in Bihar and other parts of the country. In Fig. 5 has shown the image of white fungus. Dr. Suresh Kumar, medical director of Delhi's LNJP Hospital, said on Saturday that the white fungus is not as dangerous as a black fungus. That happens later. For treatment. May persist for $1-1.5$ months so early diagnosis is important [6]. This came after many medical practitioners had previously claimed that the white fungus was more dangerous than the black fungus. Explaining how to avoid fungal infections, he said, "Fungus thrives in tight and humid places, so make sure your surroundings are cleaned regularly. Avoid refrigerated food for a few days, eat fresh fruits, keep your home Let sunlight in and wash your mask daily."

Talking about black fungus he further said, "LNJP has 12-13 patients with black fungus, most of these patients have type 2 diabetes. They took steroids continuously for 20-22 days. People should take steroids only then When prescribed by doctors. Steroids should not be used for more than 10 days during COVID treatment." A few days ago, after the first four white 


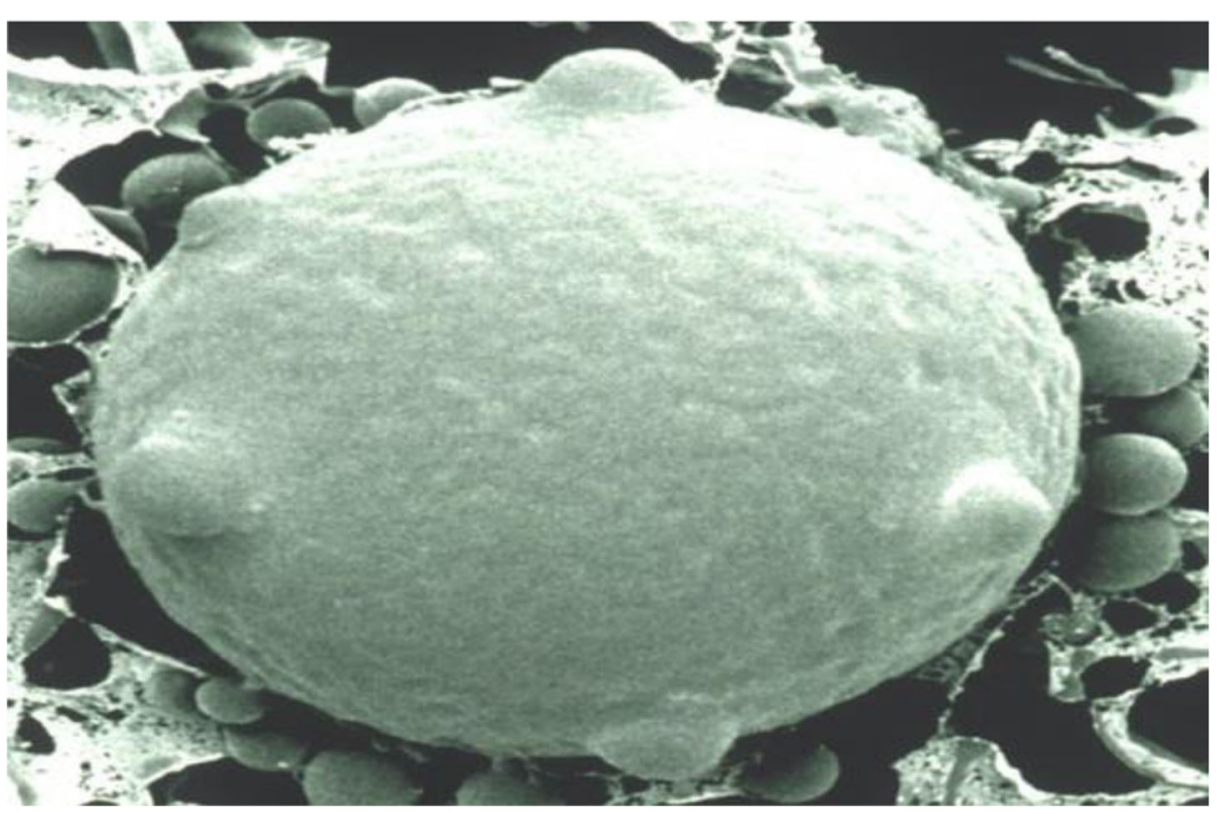

Fig. 5 White fungus [23]

fungus cases were reported in Bihar, Dr. SN Singh, head of microbiology at Patna Medical College and Hospital (PMCH), explained that people who are infected with white fungus showed a coronavirus-type has gone. Symptoms but they were not COVID patients. He also said that like black fungus, white fungus is also more dangerous for diabetic patients and those who have taken steroids for a long time [7].

\subsection{Almost every organ is affected}

In medical language, it is called Candida, which affects every part of the body through the blood. It can infect nails, skin, stomach, kidney, brain, private part and mouth as well as lungs. However, the patients who are coming affected by this fungus are not necessarily infected with Covid. However, due to the effect on the lungs, their symptoms are almost similar to corona, such as shortness of breath or sometimes chest pain. If the infection affects the joints of the body, then pain starts in them. If it reaches the brain, then there is an effect on the ability to think. The patient is not able to take decisions quickly and there is a problem in speaking also. Apart from this, there may be vomiting along with severe pain in the head. Small boils can occur when the skin spreads through blood, which are usually painless. This is an early symptom of infection. Many times people consider themselves to be corona infected without investigation and start taking medicines at home, due to the effect of corona on the lungs, it worsens the situation. The infection engulfs the main organs of the body and the patient can also die due to organ failure [32].

\subsection{Reason behind to this dangerous fungal infection}

People who have weak immunity can get this infection if they come in contact with infected flora or contaminated water. Apart from this, severe Covid-infected patients, 
who are being given oxygen, can also get the infection, if the equipment on the nose or mouth is fungal. Apart from this, its risk is more in people who are diabetic patients, or are taking steroids for a long time. Apart from these, women are also more prone to white fungus and it is seen in them in the form of leucorrhoea i.e. white discharge from genitalia. Cancer patients are also more afraid of this infection. If the patient gets RTPCR done after showing symptoms like corona infection and the test result comes negative, then experts advise him to get High-resolution computed tomography (HRCT) done for corona. In this, the lungs look like spheres, which is different from the corona. Then patients are examined for sputum culture, in which it is confirmed [41].

\subsection{Treatments for white fungus}

Treatment begins with anti-fungal drugs. However, medicines are more effective only when the disease is caught in the early stages. If detected late, the condition of the patient can also become serious, then the treatment is decided accordingly.

\section{Yellow fungus: More fatal than that of Black and white fungus}

New diseases are emerging one after the other in the patients who are in the grip of the second wave of corona virus. Initially some people became diabetic patients after getting infected with it and some were caught by Covid pneumonia. In the past, many corona infected died of heart attacks and in recent times the black fungus has wreaked havoc in a tremendous way. White fungus came after black and now yellow fungal infection has also come [6].

According to media reports, yellow fungus has been confirmed in a hospital located in Ghaziabad, UP. Yellow fungus infection has been found in a 45-year-old man. This disease is called Mucker spectics. Come, know what is the yellow fungus and how different its symptoms are from both the previous fungal infections? According to experts, the symptoms of yellow fungus are different from both black and white fungal infections, which start from the internal parts of the body. This infection is formed due to the leakage of pus in the body. Its symptoms are different from the other two fungal infections. But as it grows inside the body, it takes a deadly form. After coming under the grip of this infection, if someone has any wound, then pus starts leaking from it and the wound heals very slowly. During this, the patient's eyes get sunken and many organs stop working. His metabolic system also gets disturbed. In Fig. 6 has shown the image of yellow fungus [7] [33].

\subsection{Reptiles do not survive being hit by the yellow fungus}

Ghaziabad ENT surgeon Dr. BP Singh told the media that till now the yellow fungus was found in creatures like lizards and chameleons. According to the doctor, the reptile who has this fungus does not survive. Now it can be estimated from this how dangerous this fungus can be for humans [40]. Therefore, whenever you feel the above symptoms, get your treatment immediately without delay. The doctor says that for the first time this fungus has been found in a human. 


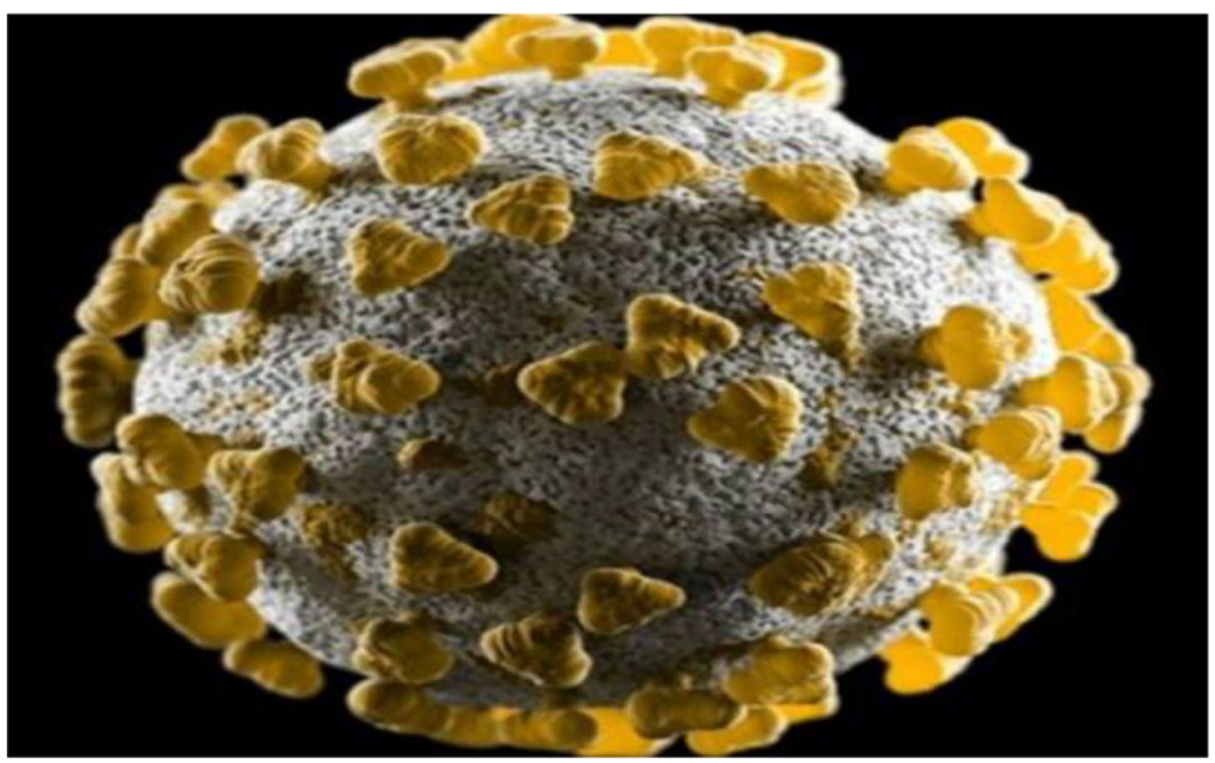

Fig. 6 Yellow fungus [33]

\subsection{Yellow fungus be more hazardous than Black or white fungus}

In the midst of the deadly corona virus epidemic, India is currently battling with other dangerous diseases caused by it. Cases of black fungus and recently white fungus are increasing in many states of the country, both the infections are spread due to fungal and unhygienic conditions present in the environment. So far, more than eight thousand cases of these infections have been reported in India. However, apart from these two infections, now a third infection is also being seen. After black and white fungus in Uttar Pradesh, now the case of yellow fungus has also come to the fore. Because of the way the yellow fungus infection affects the body's interior organs, experts believe it is considerably scarier than the other two infections. Unlike the other two diseases, yellow fungus starts inside the body, causes pus to flow out, wounds to heal slowly, and in severe cases, organ failure and acute necrosis can occur. Therefore, as soon as the patient starts showing symptoms, he should immediately seek medical help without losing wax [104].

\subsection{The main reason for the spread of this fungus}

It is important to know that most fungal infections of this type are triggered by unhygienic conditions are poor hygiene, contaminated resources (including food), or excessive steroid use, antibacterial drugs or poor oxygen use. Patients who already have a serious illness and those who are taking immunosuppressant drugs are at an increased risk of this type of infection.

\subsection{Symptoms of yellow fungus}

The symptoms of black and yellow fungus infection are disturbing. Due to which the face can be disfigured and there can also be terrible swelling. Yellow fungus is more dangerous than 
these two because it starts spreading from inside the body and can cause a variety of symptoms in the beginning.

i. Exhaustion- This fungal infection starts spreading inside the body, due to which there is pressure on the organs and you feel terrible tiredness. This can lead to a feeling of intense lethargy and tiredness.

ii. Loss of appetite- Due to the spread of yellow fungus in the body, your digestion can also be disturbed. Suddenly the patient starts showing symptoms like loss of appetite.

iii. Weight loss, poor metabolism- Metabolic changes are also a symptom that needs to be monitored at this time. Experts believe that even if there is sudden weight loss, a person must get tested. Especially if there are other symptoms associated with fungal infection.

iv. Sunken eyes- Discoloration of the face is a characteristic symptom of black fungus. Experts believe that in some severe cases of yellow fungus, the patient has to go through difficulties such as reddening of the eyes and sunken appearance, slow healing of wounds and eventually necrosis. At the same time, in some severe cases, pus can also be seen.

\subsection{Treatment of fungus (Black, white, yellow)}

Yellow, black or white fungus is not a new or unusual type of fungus. These already exist. At this time amphotericin B injection, which is an antifungal medication, is the only known treatment for fighting the infection.

\section{Aspergillosis fungal infection}

India was still battling the deadly wave of corona virus that many other types of fungal infections have increased everyone's concern. So far, a total of 12,000 cases of black fungus have been reported across the country. Gujarat and Maharashtra are the states which have been most affected by this infection. Cases of black fungus are increasing continuously in Gujarat, meanwhile a new type of fungal infection has knocked before the doctors of Vadodara. Now another fungus has started spreading. Which is called aspergillosis. After black, then white and then yellow fungus, now cases of fungus named Aspergillosis have also been reported. Aspergillosis fungus, like black fungus, is seen in persons who have just cured from Covid19. On Thursday, 262 new cases of black fungus and eight cases of aspergillosis were reported in Vadodara. All these 8 patients have been admitted to the hospital [15].

\subsection{About aspergillosis fungal infection}

Aspergillosis is a fungal infection. Aspergillosis is an infection caused by a type of mold (fungus). In Fig. 7 has shown the image of Aspergillosis Fungal. Illnesses caused by aspergillosis infection usually affect the respiratory system, but their symptoms and severity can vary [7]. The mold that triggers diseases, Aspergillus, is present everywhere indoors and outdoors. This fungus is present only in the environment around us, but it does not harm those whose immunity is strong. However, through the breath of weak people or a person with lung infection, it causes allergies by going inside the body. Its severity can also be understood from the fact that it can spread to the blood vessels and beyond. This aspergillosis affects the body in 


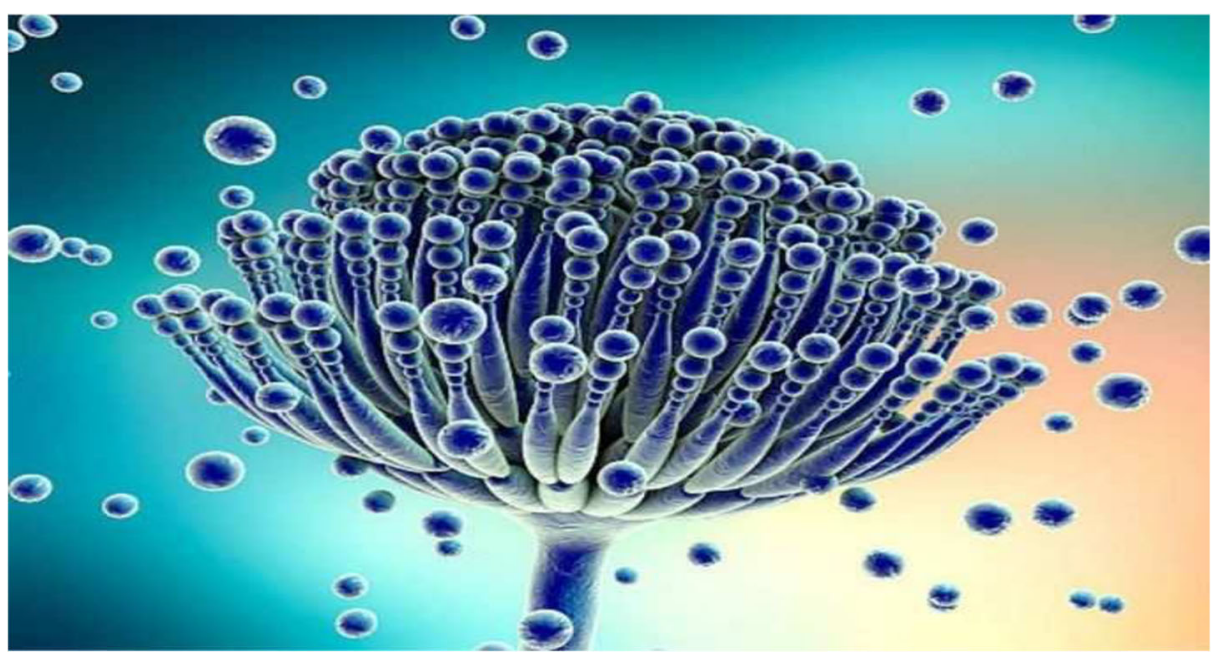

Fig. 7 Aspergillosis fungal [5]

different ways [56]. Most people inhale Aspergillus spores, but they do not get sick. Persons with a compromised immune system or lung problems, on the other hand, are more likely to acquire Aspergillus-related medical issues. Aspergillosis is commonly seen in people who have weak immunity. However, sinus pulmonary aspergillosis, which is currently being observed in COVID patients, is rare. Experts believe that aspergillosis is not as deadly as the black fungal infection, but can prove to be fatal at times.

\subsection{Effect on human life}

Steroids and weak immunity are believed to be the reason behind many fungal infections occurring in Covid-19 patients. Also, the reason behind this is being told not to use clean water to hydrate the oxygen supply. The overuse of steroids in the treatment of COVID-19 is one of the main reasons behind the increasing cases of black fungus. This is the reason why health experts have been warned against excessive use of steroids. In Fig. 8 has shown that how aspergillosis Fungal present in human lungs [56].

\subsection{Symptoms of aspergillosis infection}

i. Difficulty in breathing- If you are having trouble in breathing then it can be a sign of infection. After reaching the lungs, this fungus starts damaging the tissues. Which causes difficulty in breathing. Understand that the infection has reached the lungs [15].

ii. Fever and chills- The initial symptoms of corona are also fever and chills. In such a situation, even after you have been completely cured of corona infection, you still get fever, then it can be a sign of fungal infection.

iii. Coughing up blood- If this infection has reached your lungs, then you will have a persistent cough. Some people may also cough up some blood along with the cough.

iv. Pain in the head and eyes- The fungus enters the body through the nose. It mainly causes infections in the sinuses, lungs. Then this fungus starts moving towards the brain. Due to which pain persists in the head and eyes. 


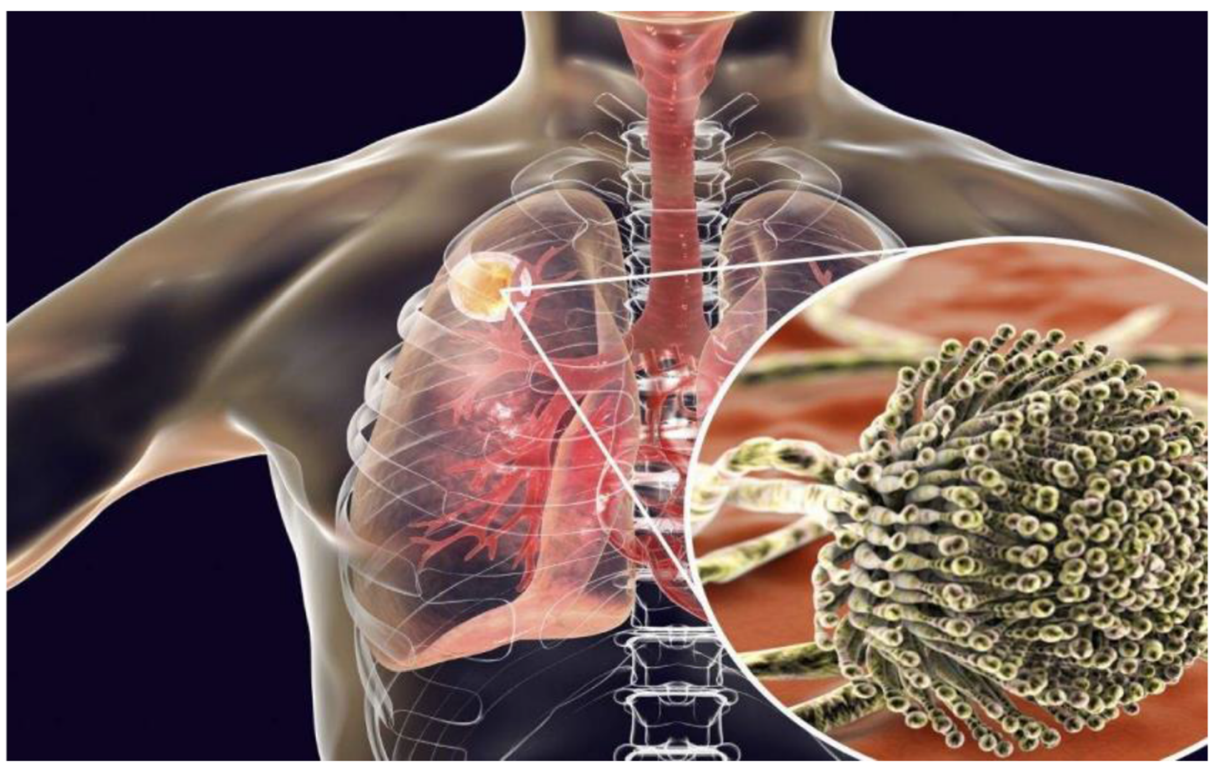

Fig. 8 Aspergillosis fungal present in human lung [5]

v. Fatigue and Weakness- Although there is a lot of weakness and fatigue after corona, but if you have any kind of infection then it increases even more. Even you find it difficult to do everyday tasks.

vi. Skin Infection- Fungal infection is affecting the skin in many cases as well. This can cause skin irritation, redness, swelling and pimples. There may also be itching all over the skin.

vii. Diagnosis of Aspergillosis- The diagnosis of Aspergillosis is a bit difficult, although doctors identify it after doing biopsy, blood test, chest X-ray, CT and lung scan.

\subsection{Treatment of aspergillosis}

The sooner it is detected about any fungal infection, the better. According to health experts, it is also treated like black and white fungus. Surgery also has to be done if the infection spreads more in the body. According to media reports, eight patients have been found infected with the new fungal infection at SSG Hospital in Vadodara. According to the information, like black fungus and white fungal infection, it is also catching the same people who are recovering from Covid. Aspergillosis is an infection caused by Aspergillus, a common mold infection, according to the Centres for Disease Control and Prevention. An infection produced by a form of mould is known as aspergillosis (fungus). Infections produced by Aspergillosis primarily affect the patient's respiratory system, but the symptoms and severity of the illness might vary. Aspergillus, the diseasecausing mould, can be found both indoors and outdoors. In this infection, Aspergillus spores enter the body of most people through breathing, although they do not get sick, but the immune system has a big role in this too. This fungus can also infect the gastrointestinal tract (gastrointestinal tract) [7]. 


\section{Various fungus diagnosis by computer vision techniques}

Artificial intelligence is a technical system in which the ability to think, understand, act and react like humans is developed through software. It has the ability to think, understand and learn in an artificial way. Although people consider robots to be artificial intelligence, whereas artificial intelligence is inserted in robots $[3,8,42]$. It would be great to use artificial intelligence in the public health sector to tackle staffing shortages and enhance the quality of treatment [59]. It is generally not easy to detect any type of fungus. But still scientists have found many types of techniques, due to which we can easily detect fungus in objects or humans. But all those techniques like hog feature pay based and SIFT (scale invariant feature transform) based techniques are very time consuming and very difficult to detect as well as very expensive. But in today's era, through computer vision (Artificial Intelligence [51, 58], Machine Learning [39], Data Learning [10, 13, 24, 25]) everything has become easy and simple. Talking about computer vision, it is a very fast and vast technology. This technique will make it much easier to locate the fungus in a patient with black, white, or yellow fungus $[6,7]$. As we know that due to covid there is a lot of outcry in the whole world. So it would be natural that everyone is very sad because of this. But the sorrow is not over yet that a new lifethreatening disease, which is popularly known as Black Fungus, has made life difficult for the people. So it has become very important to find a cure for this life-threatening fungus by taking the help of our today's devices and technology such as artificial intelligence, data learning. So there's actually a lot of sense in using artificial and machine learning to help with the treatment of fungus [52]. If we talk about any image, then there are many steps in it to read the image like image application, segmentation, feature extraction, classification $[18,21,22]$.

\subsection{Pre-processing}

So all these have different types of subparts like in the first step pre-processing, there is a lot of requirement for smoothing the image because the quality of the image also determines the result. So 2D Gaussian Filter can be used in Image Smoothing [64]. Then we come to the image enhancement, then in this process the fungus which is in the image in full quantity, they get highlighted. In Fig. 9 we show some techniques of enhancement the image of fungus like $[36,61]$.

\subsection{Segmentation}

Image segmentation is a crucial step in the analysis of medical images. Image segmentation is an important pre-processing step in analysis and classification purposes [2]. Its primary purpose is to separate the region of interest (ROI) from the surrounding environment. It also allows for the extraction of essential properties such as tissue textures and form [48]. Medical images can now be employed extensively in a variety of medical treatments, thanks to recent advancement in the field of medical imaging. As a result, each day, massive amounts of healthcare photos are produced. With such a large number of photos, analysing and diagnosing becomes a major difficulty, as manual segmentation takes time and may not be able to keep up with the need for processing large amounts of data [2].

Developing automatic algorithms for medical image segmentation is a critical and pressing subject in this regard. As a result, many authors have explored many medical image 
Grayscale image

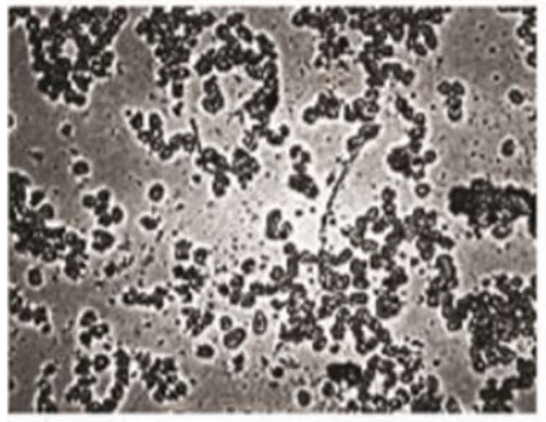

\section{Contrast stretching image}

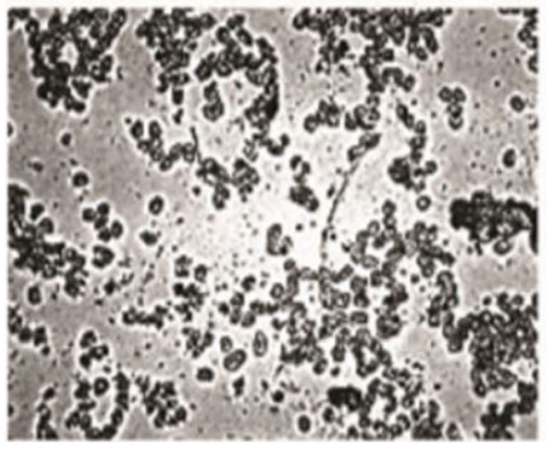

Histogram equalization image

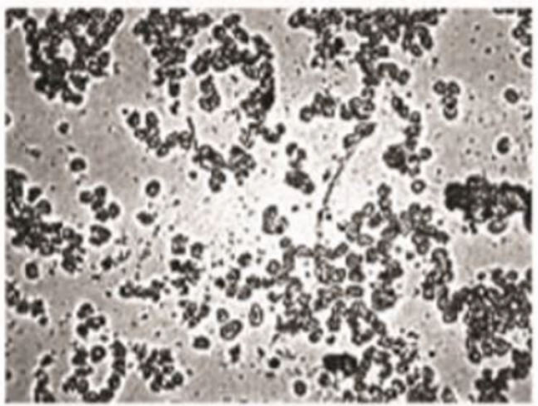

Adaptive Histogram equalization image

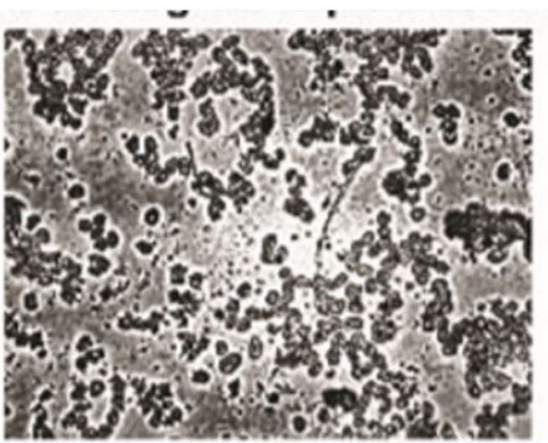

Fig. 9 Different types of image enhancement [47]

segmentation approaches employing diverse technologies in past few decades, such as clustering methods, deep learning, threshold algorithms, region-based methods, machine learning techniques, and others. In Computer-Aided Diagnosis (CADx) methods, segmentation of Computed Tomography (CT) images is a significant stage. As a result, numerous research have been suggested, such as Dev et al. [37], who advocated utilising the support vector machine (SVM) approach to diagnose lung cancer from DICOM CT-Scans. The visuals that were evaluated were either malignant or non-cancerous. Shakeel et al. [78] used a PCT to segment lung CT pictures and then used a deep learning method to identify lung tumours from the tested CT images. Medeiros et al. [68] introduced a lung CT image segmentation approach which is based on the active contour method (ACM) and a fuzzy boundary detector. Wang et al. [97] proposed an adaptive fully dense (AFD) neural network-based CT image segmentation approach. CT pictures of liver cancer were used to test their suggested approach. They also demonstrated that this approach can segment CT images with complex borders. Using a multi-depth fusion network, Ye et al. [103] suggested a method for segmenting cardiac CT images. Regarding lung CT image segmentation, Paulraj et al. [73] suggested a probabilistic fuzzy C-means technique. For object recognition in lung nodules, Han et al. [46] applied generative adversarial networks (GANs). Chen et al. [26] introduced a dictionary-based technique for segmenting abnormal 3D CT images of the lungs instantly. A numerical approach for lung CT image segmentation was suggested by Swierczynski et al. [90]. The 
suggested level-set concept merges ChanVese segmentation with active dense displacement prediction [2].

Black fungus has received a lot of interest recently because this is a new type of fungus that is very contagious and has never been observed in humans earlier. Effective segmentation algorithms could be used to automatically segment the infected zone. Numerous segmentation methods have been reported in various studies that have produced high reliable and effective segmentation outcomes [9]. Clustering-based [77], graph-cut-based [60], neural-networkbased [50], and active-contour-based approaches [105] are the four broad categories in which they can be categorized. Clustering-based solutions include clustering algorithms such as K-means and fuzzy C-means, which are associated with the concept where each image pixel may be attributed to pixels belonging to the same class based on their distribution. Clustering-based approaches are efficient, though they are sensitive to the initial clustering centres and require human clustering amount tuning. The pixel's correlation is taken into consideration in the graph-cut-based approaches, and the segmentation issue is changed into a graph partition problem, where a cut energy model is developed, and the segmentation curve is the split that minimises the energy. The problem of establishing appropriate weights for the correlations between pixels is a downside of this method, which frequently leads to over-segmentation or undersegmentation issues, particularly in dense regions. The rise of neural network-based semantic segmentation solutions necessitates a huge number of images to build the connection, and it also lacks precise segmentation for small places, which is critical in medical image analysis. Active contour (snake) models (ACM) are one of the strategies that consistently perform well [68]. This approach provides closed borders and has shown to be popular and commonly used. The basic idea underlying ACMs is to use partial differential equations (PDEs) to repeatedly evolve an initial contour near to the object boundaries by minimising an energy functional [57] that is a factor of the contour's internal and external energies. Initialization and convergence are two major issues in ACM applications. The ORACM is developed as a method to express the set level with ACM based on regions. There are no parameters in the design. When contrasted to typical ACMs, the ORACM takes less time while maintaining the same level of segmentation accuracy. ORACM2 (Online region-based active contour model with morphological operations) provides the best overall outcomes in terms of accuracy and speed, according to [9]. It must be emphasised that in COVID-19 scenarios, segmentation techniques can be split into two sections: lung-region-oriented and lunglesion-oriented methods. In CT or X-ray, lung-region-oriented approaches try to differentiate lung portions, such as the complete lung and lung lobes, from other (background) portions, which would be regarded a pre-requisite step in COVID-19 usage [107] which can be also use for Mucormycosis. Classic U-Net [107], UNet++ [62], and VB-Net [79] are some of the most common segmentation networks for fungus. In the fungus pictures, UNet and its derivatives obtained satisfactory segmentation accuracy. Jin et al. [54], for instance, employed the UNet++ network to segregate lung regions from CT images. After that, the lung's damaged region is separated. Because the amount of damage may have a range of forms and textures, this procedure might be difficult. [44] used a deep learning strategy to categorize CT scans into coronavirus and non-coronavirus patients using an autonomous AI-based study. They utilised UNet to segment lung pictures and repurposed a technique that had previously been used to detect tiny opacities and nodules within the lung [9]. 


\subsubsection{Chest X-ray}

Nashik-based company ESDS's AA+ Covid-19 Testing Solution can find out whether the patient has been suffering from Covid-19 till 5 min ago. It is a completely contactless verification process. In this process, the health workers just have to take a chest X-ray and upload the film or photo on their web browser and click on the submit button [1, 10]. After clicking on the submit button, it will instantly know whether the patient is suffering from COVID-19 or not. It has $98 \%$ accuracy. So we can also use it for epidemics like Black, White, Yellow, and Aspergillosis fungus. However, this has not been proved yet [18]. The picture of the bones and organs of the body appears black or white because of the absorption of X-ray radiations in different amounts in different tissues. Bones appear white in X-rays because the calcium in the bones absorbs a large amount of radiation [14]. The lungs hold air and the air absorbs less radiation, which makes the lungs appear black. Like in Fig. 10a and b has shown $\mathrm{X}$-Ray of Human normal human lungs and X-Ray of Human lungs which is effected by Black fungus. Also in Fig. 11a and b shows the X-Ray of normal Human lung and X-Ray of lungs which is effected by Aspergillosis fungus. X-ray Images are a greater number of people are able to access it from all over the world in comparison with CT images. Moreover, because ribs are placed onto soft tissues in $2 \mathrm{D}$, causing image contrast to be muddled, segmenting Xray pictures becomes even more difficult. Despite the facts and to the best of our knowledge, none of the image segmentation methods for fungus are used on X-ray images [9, 102].

\subsubsection{CT-scan}

CT scan is Computerized Tomography Scan. This is a type of three dimensional X-ray [17]. Tomography means to study anything by cutting it into small sections. In the case of Covid, the CT-Scan that doctors conduct is HRCT Chest that is High Resolution Computerized Tomography Scan of the chest. Through this test, the lungs are seen in 3D images. Due to this, lung infection is detected early. Although doctors say that do not go for CT scan without doctor's advice or it should not be done at all without symptoms. In hospitals, a thoracic computed tomography (CT) examination is used as some diagnostic tool lung infections.
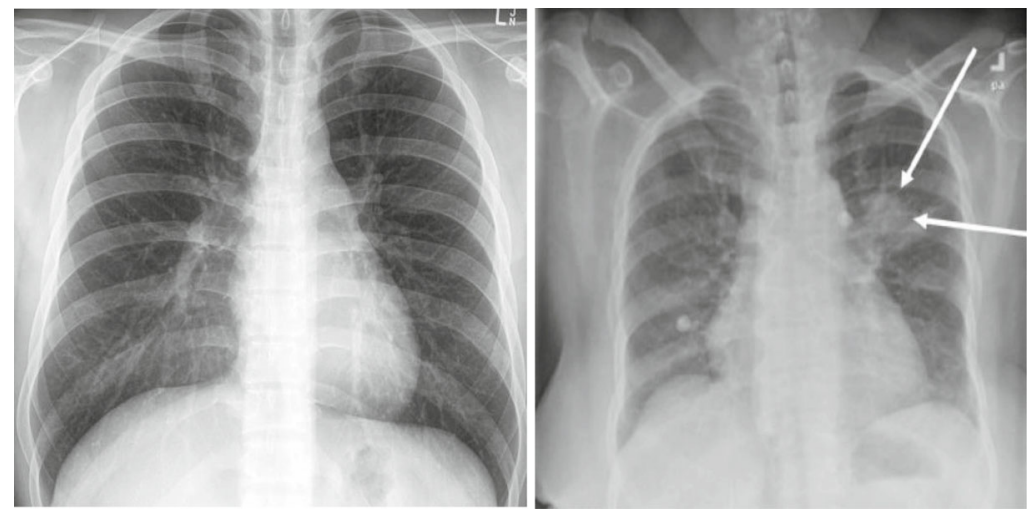

(a) X-Ray of Human normal human lungs (b) X-Ray of Human lungs which is effected by Black fungus [10]

Fig. 10 a X-Ray of human normal human lungs, $\mathbf{b}$ X-Ray of Human lungs which is effected by black fungus [12] 


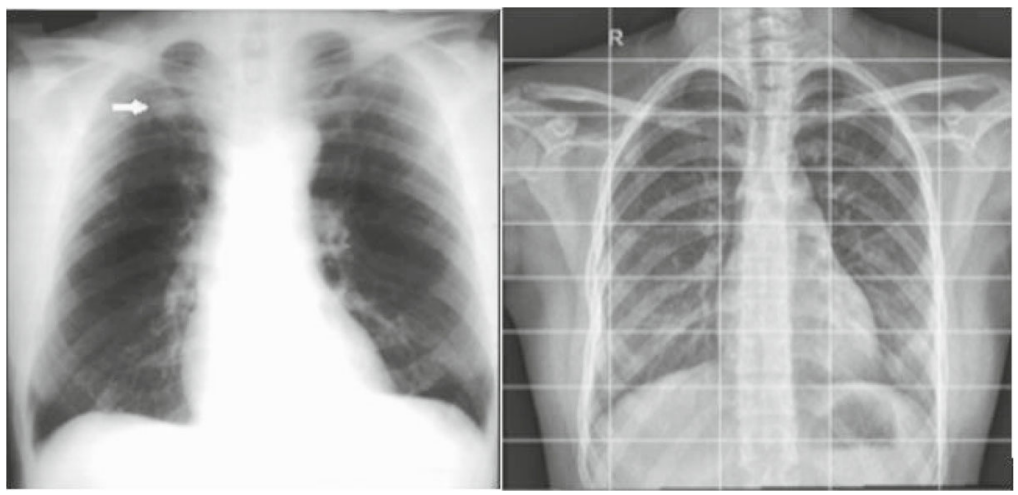

(a) X-Ray of normal Human lung (b) X-Ray of lungs which is effected by Aspergillosis fungus [58]

Fig. 11 a X-Ray of normal human lung, b X-Ray of lungs which is effected by aspergillosis fungus [56]

Developing algorithms for CT image analysis is the current hot research aims to contribute to the automatic detection of the coronavirus as well as fungus. It is also suggested that algorithms that were applied to identify lung cancer and lung collapse using X-ray images will also be useful for identifying abnormal cases from patients [9]. Actually, it is very important to take special care of health at this time [17]. The number of victims of this wave is so high that from investigation to treatment, common people are facing a lot of difficulties. Meanwhile, CT scan is also being used loudly in the investigation of corona and fungus. AIIMS Director Dr. Randeep Guleria had said that after analysing a data of radiation, it is found that people are getting CT scan in three-three days which is not at all good for their health. Like in Fig. 12a and b has shown CT-Scan of Normal lungs (b) CT-Scan of lungs which is effected by Aspergillosis fungus. Also in Fig. 13a and b shows the CT-Scan of human intestine which is not effected by Black fungus (b) CT-Scan of Human intestine which is effected by Black fungus. The CT has much more adequate information and delivers greater evaluation validity than the chest X-ray, this study only used the CT for the assessment [9].
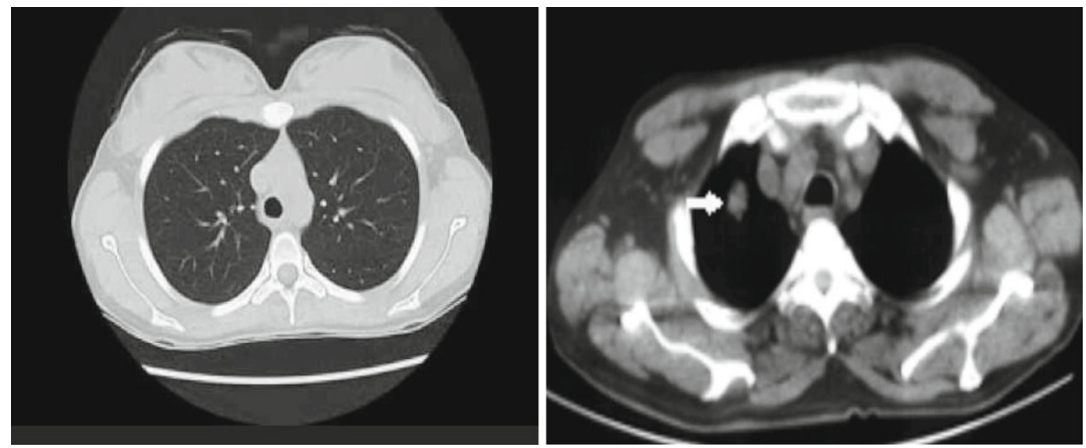

Fig. 12. (a) CT-Scan of Normal lungs [21] (b) CT-Scan of lungs which is effected by Aspergillosis fungus [15]

Fig. 12 a CT-scan of normal lungs [21], b CT-scan of lungs which is effected by aspergillosis fungus [15] 

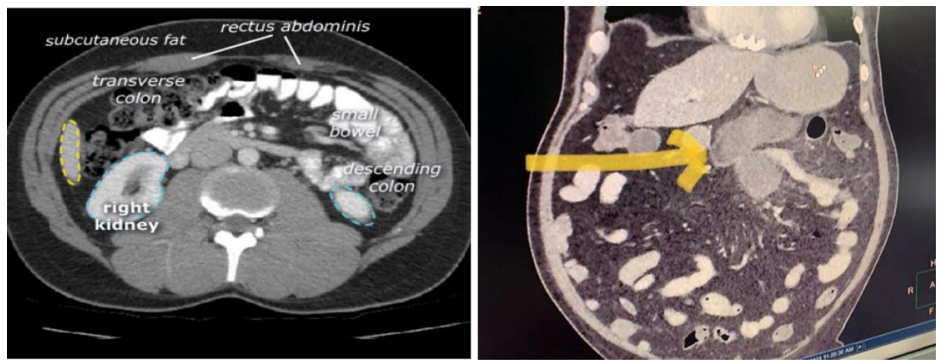

(a) CT-Scan of Human intestine which is not effected by Black fungus [21] (b) CT-Scan of Human intestine which is effected by Black fungus [21]

Fig. 13 a CT-scan of human intestine which is not effected by black fungus [21], b CT-scan of human intestine which is effected by black fungus [21]

\subsection{Feature extraction}

It was a difficult effort to extract characteristics from images of samples collected in air. There is a method for reducing the number of dimensions. The relevant features are extracted from a huge dataset using this method. Some of them are taught based on certain input features. Large data sets contain a lot of consistent and redundant data, thus extracting characteristics from them is difficult. When there's a chance of making a lot of mistakes, finding the proper input feature becomes extremely tough. However, with the help of Feature Extraction, selecting the appropriate input data becomes a breeze. The rest of the data is not included in the features since only relevant and correct input data is selected. The Machine Learning Model obtained by processing the right input data selected in Feature Extraction is of high accuracy [76].

With little modifications in lighting, these images looked significantly different $[73,75]$. Then there was the issue of anarchy. The images include a large number of fungus spores. Limiting was one of the issues with the images. The fungus detection system required a powerful feature extraction method in order to deal with all of these difficult difficulties. Dalal et al. [35] presented Histogram of Oriented Gradients as a method for detecting persons (HOG). The extraction of features from photos was accomplished using this methodology. HOG was chosen above other feature extraction approaches such as Scale-invariant feature transform descriptors (SIFT) because to its simplicity and speed of implementation [37-42]. Deep-learning-based methodologies were utilised to identify COVID -19 and normal (healthy) chest X-ray pictures in this [52] paper, including deep feature extraction, fine-tuning of pretrained convolutional neural networks $(\mathrm{CNN})$, and end-to-end training of a built CNN model. Deep CNN models (ResNet18, ResNet50, ResNet101, VGG16, and VGG19) were employed to extract deep features. The accuracy score for the deep features retrieved from the ResNet50 model and SVM classifier using the Linear kernel function was $94.7 \%$, which was the highest of all the findings.

The researcher compares and contrasts various deep learning-based feature extraction approaches for automatic COVID-19 classification in this [55]. NASNet MobileNet, Xception, InceptionResNetV2, ResNet, VGGNet, InceptionV3, and DenseNet were picked from a pool of deep convolutional neural networks to achieve more accurate characteristic, which is a key component of education. With 99\% classification accuracy, the DenseNet121 feature extractor with Bagging tree classifier achieved impressive results. With an accuracy of $98 \%$, the secondbest learner was a hybrid of the ResNet50 feature extractor trained by LightGBM. 


\subsection{Classification}

The goal of image classification is to determine and depict the features in an image in terms of the object or kind of land cover these features actually represent on the ground as a unique grey level (or colour). The most significant aspect of digital image analysis is image classification. It's lovely to have a "beautiful picture" or image with a rainbow of colours indicating different aspects of the underlying environment, but it's useless unless you know what the colours mean. PCI (PCI, 1997) Supervised Classification and Unsupervised Classification are the two main classification methods. We locate samples of the Information classes (i.e., land cover type) of interest in the image using supervised classification [65]. These are referred to as "training locations". After that, the image processing software system is utilised to create a statistical characterization of each information class's reflectance. Unsupervised classification is a technique that analyses a large number of unknown pixels and separates them into groups based on the picture value's natural groupings. Unsupervised classification does not require analyst-specified training data, unlike supervised classification. The main idea is that in the parameter space, values within a given cover type should be near combined (i.e., have comparable grey levels), while data collected from different classes should be fairly well spaced [65]. Cortes et al. [85] were the first to introduce the SVM method, which has shown to be highly effective and successful for detection and classification tasks. This method is typically used for pattern recognition. In this study, SVM is used to categorize particles as fungal spores or dirt [92]. To extract the characteristics, the [17] study uses SVM classification. During the classification algorithm, 10-fold, 5-fold, and 2-fold cross-validations were used. The classification performance was assessed using F-score, precision, sensitivity, accuracy, and specificity measures. Convolutional neural network $(\mathrm{CNN})$ demonstrates its effectiveness in pattern recognition and analysis of images. CNN has grown in popularity in the computer vision field, speech recognition, and visual analysis [91, 100]. For COVID-19 categorization from CT scans, Barstugan et al. [55] developed a machine learning approach. From $150 \mathrm{CT}$ images, patches of various sizes $(16 \times 16,32 \times 32,48 \times 48,64 \times 64)$ were identified. The algorithms Discrete Wavelet Transform (DWT), Grey Level Co-occurrence Matrix (GLCM), Grey Level Run Length Matrix (GLRLM), Local Directional Pattern (LDP), and Grey-Level Size Zone Matrix (GLSZM) were used. On 2-fold, 5-fold, and 10-fold cross-validations, the retrieved characteristics were fed into a Support Vector Machine (SVM) [34] classifier. GLSZM feature extractor using 10-fold cross-validation had the highest accuracy of $98.77 \%$.

\subsubsection{Deep belief network (DBN)}

A DBN approach has been presented in deep learning theory to collect the deep characteristics of imaging spectrum image data [49, 61]. First, using unsupervised learning techniques and a restricted Boltzmann machine (RBM), the actual data is assigned to feature region. A set of $\mathrm{RBM}$ will be used to construct a DBN, and the parameters of the model would be trained layer - by - layer by using greedy approach $[47,75]$. DBN have lately demonstrated outstanding results on a variety of classification issues. The creative qualities allow for a deeper knowledge of activities and make sensor fusion jobs easier. DBNs, on the other hand, are costly to implement on serial processors due to their intrinsic required for response and concurrent updating of huge amount of units [71]. Black fungus remedies can be detected by using DL techniques. The linked weight vector employs the manifolds neural network layers $[64,89]$. 
DL is used in a variety of applications, including computer vision, object recognition, and voice search [18, 39, 100]. AI approaches have produced many DL-based solutions for Covid19 [93]. Table 1 makes use of the literature on the AI diagnosis of COVID-19. In which we can see that many researchers have studied using different types of techniques (CNN, ResNet, $\mathrm{RF}$, and so on). However, this study is of Covid patients and because of Table 1, it can be said that the accuracy rate has come up to a great extent by using AI [8]. We can also see that ResNet50 gives quite good result as compared to other techniques used by authors. So far no such study has been done for any kind of fungus, which can be a good scope in the future.

Figure 14 illustrates through a pie chart that in the 27th meeting of the government, the UN Health Minister reported that as of May 21, 2021, there were 5424 cases of black fungus in 18 states. Out of which 4556 patients were suffering from corona and $55 \%$ of those 4556 patients were diabetic patients. And in Table 2 has shown the comparison of Black, White, Yellow, and Aspergillosis fungus for various parameters.

\section{Future research}

Numerous initiatives to diagnose fungus through computer vision (machine learning, deep learning, artificial intelligence) have indeed been undertaken, but all these methods are insufficient for Black fungus confirmation. Artificial intelligence-based image acquisition proved to be successful and effective in screening. The dataset's size needs to be increased in order to be used in hospitals [18].

The requirement to fill areas for further research becomes even more pressing in light about the need for treatment protocols for a potential third COVID-19 wave, which is predicted to disproportionately impact kids, especially those with impairments. As a result, more structured research is needed to meet the additional issues offered by Mucormycosis [39].

Mucormycosis is a fungal infection that has a $50 \%$ death rate. A growing percentage of COVID-19 sufferers are contracting the virus while still in the clinic or after being discharged. Mucormycosis is substantially more likely in COVID-19 sufferers undergoing treatment, especially those who need oxygen therapy throughout their sickness. Breathing of Mucor spores by sufferers with a weak immune system causes the fungus to colonise the body, invade the host, and cause Mucormycosis. Mucormycosis is more common in people with

Table 1 Examination on AI-related diagnosis

\begin{tabular}{|c|c|c|c|}
\hline Authors & Cases & Techniques & Accuracy rate \\
\hline Wang et al. [99] & $44+$ ve, $55-$ ve & $\mathrm{CNN}$ & $82.90 \%$ \\
\hline Wang et al. [98] & $45+$ ve, $1203-$ ve & $\mathrm{CNN}$ & $83.50 \%$ \\
\hline Ying et al. [88] & $88+$ ve, $86-v e$ & ResNet50 & $86.00 \%$ \\
\hline $\mathrm{Xu}$ et al. [101] & $219+$ ve, 175 -ve & $\mathrm{CNN}$ & $86.70 \%$ \\
\hline Tang et al. [94] & $176+\mathrm{ve}$ & $\mathrm{RF}$ & $87.50 \%$ \\
\hline Shi et al. [83] & $1658+v e, 1027-v e$ & $\mathrm{RF}$ & $87.90 \%$ \\
\hline Ghoshal et al. [43] & 70 & $\mathrm{CNN}$ & $92.90 \%$ \\
\hline Zhang et al. [106] & $70+$ ve, 1008 other & ResNet & $95.20 \%$ \\
\hline Chen et al. [28] & $51+$ ve, $51-$ ve & UNet++ & $95.20 \%$ \\
\hline Zheng et al. [107] & $313+$ ve, 229 other & UNet, CNN & $95.90 \%$ \\
\hline Narin et al. [70] & $50+$ ve, $50-$ ve & ResNet50 & $98.00 \%$ \\
\hline
\end{tabular}




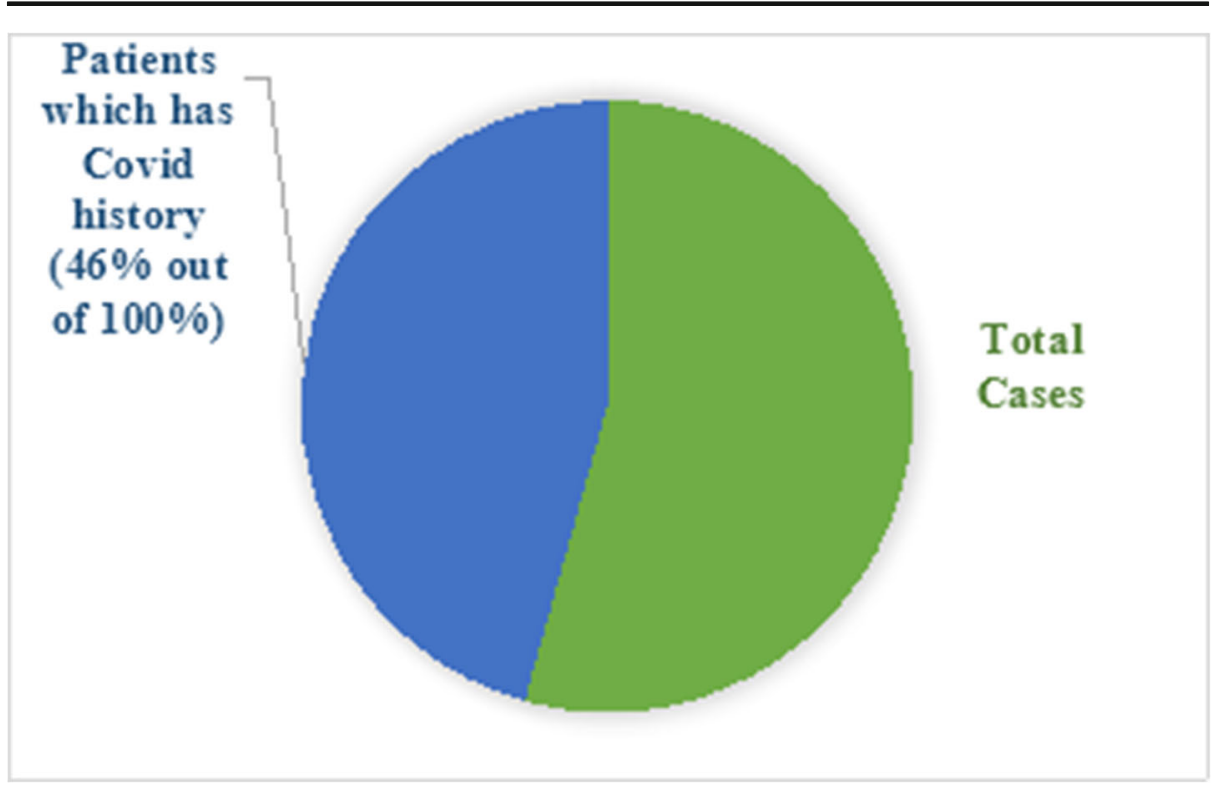

Fig. 14 Patients which has Covid history (46\% out of $100 \%$ )

uncontrolled diabetes due to high blood sugar levels make it much easier for fungi to survive and flourish. Their poor immune system provides less defence against the invading bacteria. Factors such as heat and humidity, as well as oxygen, humidifiers, and oxygen delivery masks, may all contribute to the spread of illness. The effect of this fungal infection might be made scarier if identification and therapy are delayed [17]. Figure 15 shows the impact of Covid-19 and Mucormycosis on the health of our society and people. It is a big future challenge to work on these parameters.

\section{Conclusion}

Through this paper, it is proved that Black fungus, White fungus, Yellow fungus and Aspergillosis fungus can cause so many ill effects on mankind. It usually shows its effect

Table 2 Comparison of black, white, yellow, and aspergillosis fungus for various parameters

\begin{tabular}{|c|c|c|c|c|}
\hline Parameters & Black Fungus & White Fungus & Yellow Fungus & Aspergillosis Fungus \\
\hline Colour & Black & White & Yellow & $\begin{array}{l}\text { Black on soil, and whitish or } \\
\text { Blueish underneath }\end{array}$ \\
\hline Mortality rate $(\%)$ & $50 \%$ & $40 \%$ & $60 \%$ & $30 \%$ \\
\hline Total cases in India & 12,000 & 2000 & 8000 & 1000 \\
\hline First case found & 14 May 2021 & 21 May 2021 & 24 May 2021 & 01 June 2021 \\
\hline State name & Karnataka & Bihar & Uttar Pradesh & Uttar Pradesh \\
\hline Spreading to others & No & No & No & No \\
\hline Present in soil & Yes & Yes & Yes & Not confirmed \\
\hline $\begin{array}{l}\text { Are they impact on } \\
\text { Non-Covid patients }\end{array}$ & Yes & Yes & Yes & Yes \\
\hline Dangerous & High & Medium & High & Low \\
\hline
\end{tabular}




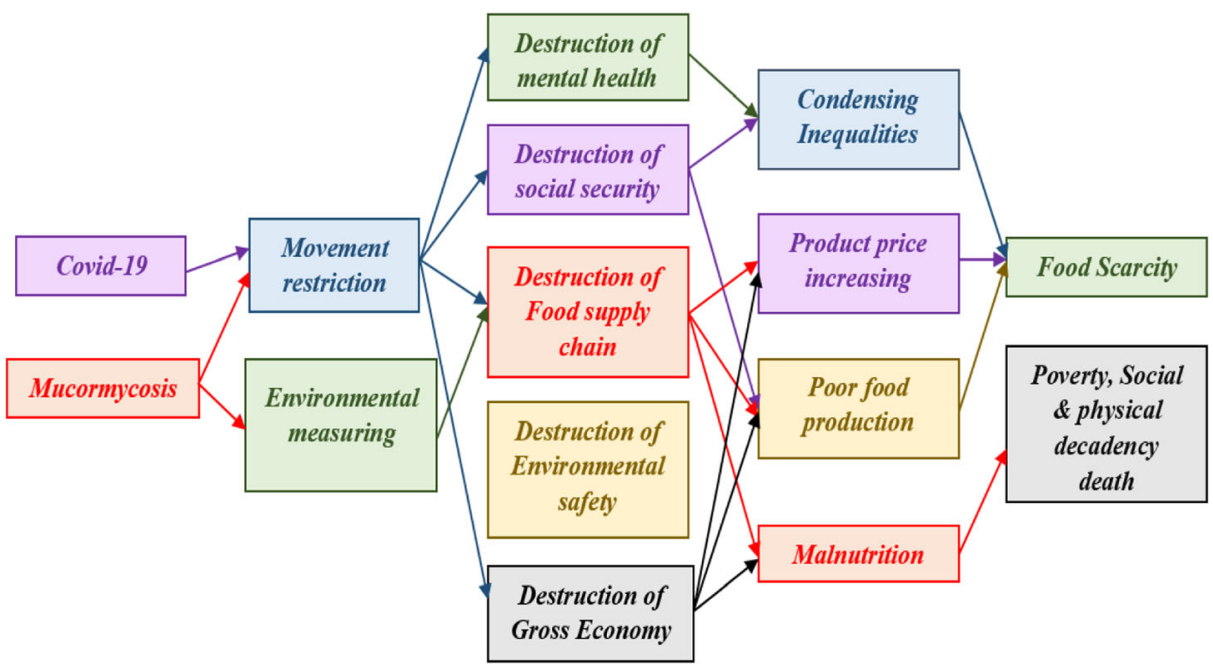

Fig. 15 Tetra outbreak tendencies (COVID-19 and Mucormycosis) pose a hazard to the social economical and community healthcare organisations. The impacts of each incident are indicated by the lines

more on people with weakened immune systems, so computer vision and image processing have been used to find a solution. Through which fungus is easily detected in the human body. Due to which it can be used rapidly in diagnosis and treatment. It has also been learned from this paper that white fungus is more dangerous than other fungi and the people who fall prey to them are either diabetic patients or their immune system is very low. A lot of information has also been given about CT-Scan and X-Ray. It was found that the CT-Scan has much more adequate information and delivers greater evaluation validity than the chest X-Ray. After that the steps for Image processing such as pre-processing, segmentation, all these were surveyed in which it was found that Deep CNN models (ResNet18, ResNet50, ResNet101, VGG16, and VGG19) were employed to extract image features. Accuracy score for the deep features retrieved from the ResNet50 model and SVM classifier using the Linear kernel function was 94.7\%, which was the highest of all the findings. Also GLSZM feature extractor using 10-fold cross-validation had the highest accuracy of $98.77 \%$. DBN has lately demonstrated outstanding results on a variety of classification issues. The creative qualities allow for a deeper knowledge of activities and make sensor fusion jobs easier. The use of another computer vision technology has yielded good results, namely DBN. DBNs, on the other hand, are costly to implement on serial processors due to their intrinsic required for response and concurrent updating of huge amount of units. It was also studied that if the diagnosis is made using technology like artificial intelligence, its accuracy gives quietly good results as compared to others technique in this pandemic.

Funding This research is not having any specific grant from funding agencies in the public, commercial, or not for profit sectors.

\section{Declarations}

Conflict of interest Authors do not have any conflicts. 


\section{References}

1. Abbas A, Abdelsamea MM, Gaber MM (2020) Classification of COVID-19 in chest X-ray images using DeTraC deep convolutional neural network. arXiv preprint arXiv:2003.13815

2. Abd Elaziz M, AA Alqaness M, Abo Zaid EO, Lu S, Ali Ibrahim R, A Ewees A (2021) Automatic clustering method to segment COVID-19 CT images. PLoS One 16(1):e0244416

3. Achanta R, Shaji A, Smith K, Lucchi A, Fua P, Süsstrunk S (2012) SLIC superpixels compared to state-ofthe-art superpixel methods. IEEE Trans Pattern Anal Mach Intell 34(11):2274-2282

4. Acharjya PP, Das R, Ghoshal D (2012) Study and comparison of different edge detectors for image segmentation. Global Journal of Computer Science and Technology

5. Adil A (2021) Over 28,200 'black fungus' cases recorded in India. Anadolu agency, 2021. https://www.aa. com.tr/en/asia-pacific/over-28-200-black-fungus-cases-recorded-in-india/2266396. Accessed 6.21.21

6. After black fungus and white fungus, another fungal infection aspergillosis, hits India (n.d.). [online]. Available: https://www.dnaindia.com/india/report-after-black-fungus-and-white-fungus-another-fungalinfection-aspergillosis-hits-india-2892150. Accessed 3 Mar 2021

7. After Black, White Or Yellow Fungus - Aspergillosis Is The New Fungal Infection Reported In Covid-19 Patients In India (n.d.). [online]. Available: https://thenationalbulletin.in/after-black-white-or-yellowfungus-ndash-aspergillosis-is-the-new-fungal-infection-reported-in-covid-19-patients-in-india. Accessed 3 Mar 2021

8. AI Helps Doctors Diagnose the Coronavirus. (n.d.) [Online]. Available: https://news.developer.nvidia. com/ai-helps-doctors-diagnose-thecoronavirus/. Accessed 3 Mar 2021

9. Akbari Y, Hassen H, Al-Maadeed S, Zughaier SM (2021) COVID-19 lesion segmentation using lung CT scan images: comparative study based on active contour models. Appl Sci 11(17):8039

10. Al-antari MA, Al-masni MA, Choi MT, Han SM, Kim TS (2018) A fully integrated computer-aided diagnosis system for digital X-ray mammograms via deep learning detection, segmentation, and classification. Int J Med Inform 117:44-54

11. Alekseyev K, Didenko L, Chaudhry B (2021) Rhinocerebral Mucormycosis and COVID-19 pneumonia. J Med Cases 12:85. https://doi.org/10.14740/jmc3637

12. Al-Tawfiq JA, Alhumaid S, Alshukairi AN, Temsah MH, Barry M, Al Mutair A, ... Dhama K (2021) COVID-19 and Mucormycosis superinfection: the perfect storm. Infection 49:1-21

13. Andreu-Perez J, Pérez-Espinosa H, Timonet E, Kiani M, Giron-Perez MI, Benitez-Trinidad AB, .... Rivas F (2021) A generic deep learning based cough analysis system from clinically validated samples for pointof-need covid-19 test and severity levels. IEEE Trans Serv Comput

14. Asnaoui KE, Chawki Y, Idri A (2020) Automated methods for detection and classification pneumonia based on X-ray images using deep learning. arXiv preprint arXiv:2003.14363

15. Aspergillosis (n.d.). [online]. Available: https://www.healthline.com/health/aspergillosis. Accessed 3 Mar 2021

16. Balachandar V, Kaavya J, Mahalaxmi I, Arul N, Vivekanandhan G, Bupesh G, Singaravelu G, Anila V, Dhivya V, Harsha G (2020a) COVID-19: a promising cure for the global panic. Sci Total Environ 725 : 138277. https://doi.org/10.1016/j.scitotenv.2020.138277

17. Barstugan M, Ozkaya U, Ozturk S (2020) Coronavirus (COVID-19) classification using CT images by machine learning methods. arXiv preprint arXiv:2003.09424

18. Bhargava A, Bansal A (2021) Novel coronavirus (COVID-19) diagnosis using computer vision and artificial intelligence techniques: a review. Multimed Tools Appl 80:1-16

19. Black fungus: Here is a list of states with highest number of Mucormycosis cases (n.d.). [online]. Available: https://www.hindustantimes.com/india-news/black-fungus-states-with-highest-number-ofMucormycosis-cases-101621559394002.html. Accessed 3 Mar 2021

20. Blank R, Vinayaka PP, Tahir MW, Yong J, Vellekoop MJ, Lang W (2016) Comparison of several optical methods for an automated fungal spore sensor system concept. IEEE Sensors J 16(14):5596-5602

21. Cao J, Jiang L, Hou J, Jiang L, Zhao R, Shi W ... Feng R (2021) Exploiting Deep Cross-Slice Features From CT Images For Multi-Class Pneumonia Classification. In; 2021 IEEE International Conference on Image Processing (ICIP) (pp. 205-209). IEEE

22. Castiglione A, Vijayakumar P, Nappi M, Sadiq S, Umer M (2021) COVID-19: automatic detection of the novel coronavirus disease from CT images using an optimized convolutional neural network. IEEE Trans Ind Inform 17:6480-6488

23. Chakraborty S, Mali K (2021) A morphology-based radiological image segmentation approach for efficient screening of COVID-19. Biomed Signal Process Control 69:102800

24. Chang V, Abdel-Basset M, Iqbal R, Wills G (2021) IEEE transactions on industrial informatics EDITORIAL: advanced deep learning techniques for COVID-19. IEEE Trans Ind Inform 17:6476-6479 
25. Chen T, Chefd'Hotel C (2014) Deep learning based automatic immune cell detection for immunohistochemistry images. In: International workshop on machine learning in medical imaging (pp. 17-24). Springer: Cham

26. Chen G, Xiang D, Zhang B, Tian H, Yang X, Shi F, Zhu W, Tian B, Chen X (2019) Automatic pathological lung segmentation in low-dose CT image using eigenspace sparse shape composition. IEEE Trans Med Imaging 38(7):1736-1749

27. Chen R, Chen J, Meng QT (2020) Chest computed tomography images of early coronavirus disease (COVID-19). Can J Anesth/J Can d'anesthésie 11:1-2

28. Chen J, Wu L, Zhang J, Zhang L, Gong D, Zhao Y, Chen Q, Huang S, Yang M, Yang X, Hu S, Wang Y, Hu X, Zheng B, Zhang K, Wu H, Dong Z, Xu Y, Zhu Y, ... Yu H (2020) Deep learning-based model for detecting 2019 novel coronavirus pneumonia on high-resolution computed tomography. Sci Rep 10(1):111

29. Cortes C, Vapnik V (1995) Support-vector networks. Mach Learn 20(3):273-297

30. Cosic I, Lazar K, Cosic D (2014) Prediction of tubulin resonant frequencies using the resonant recognition model (RRM). IEEE Trans Nanobiosci 14(4):491-496

31. COVID19 and Black Fungus: Everything you need to know about the rising Mucormycosis infections in India (n.d.). [online]. Available: https://en.gaonconnection.com/covid19-black-fungus-Mucormycosisinfections-symptoms-treatement-icmr-india/. Accessed 3 Mar 2021

32. COVID-19 India FAQs: What is White Fungus, Who It Can Infect, How It Can Be Treated and More (n.d.). [online]. Available: https://weather.com/en-IN/india/coronavirus/news/2021-05-21-covid-19-indiafaqs-what-is-white-fungus-who-it-can-infect-how-it. Accessed 3 Mar 2021

33. Covid-19: All you need to know about 'yellow fungus' (n.d.). [online]. Available: https://www. deccanherald.com/national/covid-19-all-you-need-to-know-about-yellow-fungus-989747.html. Accessed 3 Mar 2021

34. Cristianini N, Shawe-Taylor J (2000) An introduction to support vector machines and other kernel-based learning methods. Cambridge University Press

35. Dai X, Cheng J, Gao Y, Guo S, Yang X, Xu X, Cen Y (2020) Deep belief network for feature extraction of urban artificial targets. Math Probl Eng 2020:1-13

36. Dantas KC, Mauad T, de André CDS, Bierrenbach AL, Saldiva PHN (2021) A single-Centre, retrospective study of the incidence of invasive fungal infections during 85 years of autopsy service in Brazil. Sci Rep 11:1-10. https://doi.org/10.1038/s41598-021-83587-1

37. Dev C, Kumar K, Palathil A, Anjali T, Panicker V (2019) Machine learning based approach for detection of lung Cancer in DICOM CT image. In: Ambient communications and computer systems (pp. 161-173). Springer: Singapore

38. Elbossaty WF (2021) The Black fungus is one of the bad Conse-quences of COVID 19. Clin Oncol 5(2):14

39. Endres I, Hoiem D (2013) Category-independent object proposals with diverse ranking. IEEE Trans Pattern Anal Mach Intell 36(2):222-234

40. Explained: What Are Black, White And Yellow Fungal Infections, Why Do They Occur, And Precautions To Take (n.d.). [online]. Available: https://www.outlookindia.com/website/story/india-news-explainedwhat-are-black-white-and-yellow-fungal-infections-why-do-they-occur-and-precautions-to-take/383950. Accessed 3 Mar 2021

41. First case of White Fungus found in Uttar Pradesh (n.d.). [online]. Available: https://www.dnaindia.com/ india/report-first-case-of-white-fungus-found-in-uttar-pradesh-2891377. Accessed 3 Mar 2021

42. Five Companies Using AI to Fight Coronavirus. (n.d.) [Online]. Available: https://spectrum.ieee.org/ thehuman-os/artificial-intelligence/medicalai/companies-ai-coronavirus. Accessed 3 Mar 2021

43. Ghoshal B, Tucker A (2020) Estimating uncertainty and interpretability in deep learning for coronavirus (COVID-19) detection. arXiv preprint arXiv:2003.10769

44. Gozes O, Frid-Adar M, Greenspan H, Browning PD, Zhang H, Ji W, ... Siegel E (2020) Rapid ai development cycle for the coronavirus (covid-19) pandemic: Initial results for automated detection \& patient monitoring using deep learning ct image analysis. arXiv preprint arXiv:2003.05037

45. Gupta BM, Mamdapur GM, Dayal D (2021) Black fungus (Mucormycosis) research in India during 19982021: A Scopus-based Scientometric analysis. Int J Med Public Health 11(3):133-138

46. Han C, Kitamura Y, Kudo A, Ichinose A, Rundo L, Furukawa Y ... Nakayama H (2019) Synthesizing diverse lung nodules wherever massively: 3D multi-conditional GAN-based CT image augmentation for object detection. In 2019 International Conference on 3D Vision (3DV) (pp. 729-737). IEEE

47. Haralick RM, Shanmugam K, Dinstein IH (1973) Textural features for image classification. IEEE Trans Syst Man Cybern 6:610-621

48. Havaei M, Davy A, Warde-Farley D, Biard A, Courville A, Bengio Y, Pal C, Jodoin PM, Larochelle H (2017) Brain tumor segmentation with deep neural networks. Med Image Anal 35:18-31 
49. He K, Zhang X, Ren S, Sun J (2016) Deep residual learning for image recognition. In: Proceedings of the IEEE conference on computer vision and pattern recognition (pp. 770-778)

50. He K, Gkioxari G, Dollár P, Girshick R (2017) Mask r-cnn. In: Proceedings of the IEEE international conference on computer vision (pp. 2961-2969)

51. Imteaj A, Rahman T, Hossain MK, Alam MS, Rahat SA (2017) An IoT based fire alarming and authentication system for workhouse using raspberry pi 3. In: 2017 international conference on electrical, computer and communication engineering (ECCE) (pp. 899-904). IEEE

52. Ismael AM, Șengür A (2021) Deep learning approaches for COVID-19 detection based on chest X-ray images. Expert Syst Appl 164:114054

53. Jian Z, Wei Z (2010) Support vector machine for recognition of cucumber leaf diseases. In: 2010 2nd international conference on advanced computer control (Vol. 5, pp. 264-266). IEEE

54. Jin S, Wang B, Xu H, Luo C, Wei L, Zhao W, ... Xu W (2020) AI-assisted CT imaging analysis for COVID-19 screening: building and deploying a medical AI system in four weeks. MedRxiv

55. Kassania SH, Kassanib PH, Wesolowskic MJ, Schneidera KA, Detersa R (2021) Automatic detection of coronavirus disease (COVID-19) in X-ray and CT images: a machine learning based approach. Biocybern Biomed Eng 41(3):867-879

56. Khan ZU, Kortom M, Marouf R, Chandy R, Rinaldi MG, Sutton DA (2000) Bilateral pulmonary aspergilloma caused by an atypical isolate of aspergillus terreus. J Clin Microbiol 38(5):2010-2014

57. Kim W, Kim C (2012) Active contours driven by the salient edge energy model. IEEE Trans Image Process 22(4):1667-1673

58. Koley S, Ghosal P (2017) An IoT enabled real-time communication and location tracking system for vehicular emergency. In: 2017 IEEE computer society annual symposium on VLSI (ISVLSI) (pp. 671676). IEEE

59. Kumar H, Tomar VK (2020) Design of low power with expanded noise margin subthreshold 12T SRAM cell for ultra-low power devices. J Circ Syst Comput 30(06):2150106. https://doi.org/10.1142/ S0218126621501061

60. Kuo JW, Mamou J, Aristizábal O, Zhao X, Ketterling JA, Wang Y (2015) Nested graph cut for automatic segmentation of high-frequency ultrasound images of the mouse embryo. IEEE Trans Med Imaging 35(2): $427-441$

61. Lessmann N, van Ginneken B, Zreik M, de Jong PA, de Vos BD, Viergever MA, Išgum I (2017) Automatic calcium scoring in low-dose chest CT using deep neural networks with dilated convolutions. IEEE Trans Med Imaging 37(2):615-625

62. Li L, Qin L, Xu Z, Yin Y, Wang X, Kong B, Bai J, Lu Y, Fang Z, Song Q, Cao K, Liu D, Wang G, Xu Q, Fang X, Zhang S, Xia J, Xia J (2020) Artificial intelligence distinguishes COVID-19 from community acquired pneumonia on chest CT. Radiology 296:E65-E71

63. Loey M, Manogaran G, Taha MHN, Khalifa NEM (2021) Fighting against COVID-19: A novel deep learning model based on YOLO-v2 with ResNet-50 for medical face mask detection. Sustain Cities Soc 65:102600

64. Lowe DG (2004) Distinctive image features from scale-invariant keypoints. Int J Comput Vis 60(2):91110

65. Mahalaxmi I, Jayaramayya K, Venkatesan D, Subramaniam MD, Renu K, Vijayakumar P, Narayanasamy A, Gopalakrishnan AV, Kumar NS, Sivaprakash P, Sambasiva Rao KRS, Vellingiri B (2021) Mucormycosis: an opportunistic pathogen during COVID-19. Environ Res 111643:111643

66. Mahalaxmi I, Kaavya J, Mohana Devi S, Balachandar V (2021) COVID-19 and olfactory dysfunction: a possible associative approach towards neurodegenerative diseases. J Cell Physiol 236:763-770. https://doi. org/10.1002/jcp.29937

67. Maini A, Tomar G, Khanna D, Kini Y, Mehta H, Bhagyasree V (2021) Sino-orbital Mucormycosis in a COVID-19 patient: a case report. Int J Surg Case Rep 82:105957. https://doi.org/10.1016/j.ijscr.2021. 105957

68. Medeiros AG, Guimarães MT, Peixoto SA, Santos LDO, da Silva Barros AC, Rebouças EDS, de Albuquerque VHC, Rebouças Filho PP (2019) A new fast morphological geodesic active contour method for lung CT image segmentation. Measurement 148:106687

69. Mucormycosis: The 'black fungus' maiming Covid patients in India (n.d.). [online]. Available: https:// www.bbc.com/news/world-asia-india-57027829. Accessed 3 Mar 2021

70. Narin A, Kaya C, Pamuk Z (2021) Automatic detection of coronavirus disease (covid-19) using X-ray images and deep convolutional neural networks. Pattern Anal Applic 24:1-14

71. O'Connor P, Neil D, Liu SC, Delbruck T, Pfeiffer M (2013) Real-time classification and sensor fusion with a spiking deep belief network. Front Neurosci 7:178 
72. Pardeshi V, Sagar S, Murmurwar S, Hage P (2017). Health monitoring systems using IoT and raspberry pi-a review. In: 2017 international conference on innovative mechanisms for industry applications (ICIMIA) (pp. 134-137). IEEE

73. Paulraj T, Chelliah KSV, Chinnasamy S (2019) Lung computed axial tomography image segmentation using possibilistic fuzzy C-means approach for computer aided diagnosis system. Int J Imaging Syst Technol 29(3):374-381

74. Prakash H, Skiada A, Paul RA, Chakrabarti A, Rudramurthy SM (2021) Connecting the dots: interplay of pathogenic mechanisms between COVID-19 disease and Mucormycosis. J Fungi 7(8):616

75. Pujari JD, Yakkundimath R, Byadgi AS (2013) Classification of fungal disease symptoms affected on cereals using color texture features. Int J Signal Process Image Process Pattern Recog 6(6):321-330

76. Raza AF, Paudel DR, Prabhu P (2021) Black fungus and COVID-19: role of otorhinolaryngologists and audiologists. Eur Arch Otorhinolaryngol 278:1-2

77. Ribbens A, Hermans J, Maes F, Vandermeulen D, Suetens P (2013) Unsupervised segmentation, clustering, and groupwise registration of heterogeneous populations of brain MR images. IEEE Trans Med Imaging 33(2):201-224

78. Shakeel PM, Burhanuddin MA, Desa MI (2019) Lung cancer detection from CT image using improved profuse clustering and deep learning instantaneously trained neural networks. Measurement 145:702-712

79. Shan F, Gao Y, Wang J, Shi W, Shi N, Han M ... Shi Y (2020) Lung infection quantification of COVID-19 in CT images with deep learning. arXiv preprint arXiv:2003.04655

80. Shariati A, Moradabadi A, Chegini Z, Khoshbayan A, Didehdar M (2020) An overview of the management of the most important invasive fungal infections in patients with blood malignancies. Infect Drug Resist 13:2329-2354. https://doi.org/10.2147/IDR.S254478

81. Sharma S, Grover M, Bhargava S, Samdani S, Kataria T (2021) Post coronavirus disease Mucormycosis: a deadly addition to the pandemic spectrum. J Laryngol Otol 135(5):442-447

82. Shi J, Malik J (2000) Normalized cuts and image segmentation. IEEE Trans Pattern Anal Mach Intell 22(8):888-905

83. Shi F, Xia L, Shan F, Wu D, Wei Y, Yuan H ... Shen D (2020) Large-scale screening of covid-19 from community acquired pneumonia using infection size-aware classification. arXiv preprint arXiv: 2003.09860

84. Shrestha A, Mahmood A (2019) Review of deep learning algorithms and architectures. IEEE Access 7: 53040-53065

85. Simonyan K, Zisserman A (2014) Very deep convolutional networks for large-scale image recognition. arXiv preprint arXiv:1409.1556

86. Singh AK, Singh R, Joshi SR, Misra A (2021) Mucormycosis in COVID-19: a systematic review of cases reported worldwide and in India. Diabetes Metab Syndr Cli Res Rev

87. Slavin M, Thursky K (2021) Mucormycosis: the Black fungus hitting Covid-19 patients. BBC

88. Song Y, Zhang L, Chen S, Ni D, Li B, Zhou Y ... Wang T (2014) A deep learning based framework for accurate segmentation of cervical cytoplasm and nuclei. In: 2014 36th Annual International Conference of the IEEE Engineering in Medicine and Biology Society (pp. 2903-2906). IEEE

89. Song Y, Zheng S, Li L, Zhang X, Zhang X, Huang Z, Chen J, Wang R, Zhao H, Chong Y, Shen J, Zha Y, Yang Y (2021) Deep learning enables accurate diagnosis of novel coronavirus (COVID-19) with CT images. IEEE/ACM Trans Comput Biol Bioinform 18:2775-2780

90. Swierczynski P, Papież BW, Schnabel JA, Macdonald C (2018) A level-set approach to joint image segmentation and registration with application to CT lung imaging. Comput Med Imaging Graph 65:5868

91. Szegedy C, Liu W, Jia Y, Sermanet P, Reed S, Anguelov D ... Rabinovich A (2015) Going deeper with convolutions. In: Proceedings of the IEEE conference on computer vision and pattern recognition (pp. 1-9)

92. Tahir MW, Zaidi NA, Blank R, Vinayaka PP, Vellekoop MJ, Lang W (2017) Fungus detection through optical sensor system using two different kinds of feature vectors for the classification. IEEE Sensors $\mathrm{J}$ 17(16):5341-5349

93. Tan C, Sun F, Kong T, Zhang W, Yang C, Liu C (2018) A survey on deep transfer learning. In: International conference on artificial neural networks (pp. 270-279). Springer: Cham

94. Tang Z, Zhao W, Xie X, Zhong Z, Shi F, Liu J, Shen D (2020) Severity assessment of coronavirus disease 2019 (COVID-19) using quantitative features from chest CT images. arXiv preprint arXiv:2003.11988

95. Tang S, Wang C, Nie J, Kumar N, Zhang Y, Xiong Z, Barnawi A (2021) EDL-COVID: ensemble deep learning for COVID-19 cases detection from chest X-ray images. IEEE Trans Ind Inform 17:6539-6549

96. Vishnupriya M, Naveenkumar M, Manjima K, Sooryasree N, Saranya T, Ramya S, Winster SH, Paulpandi M, Balachandar V, Arul N (2021) Post-COVID pulmonary fibrosis: therapeutic efficacy using with mesenchymal stem cells-how the lung heals. Eur Rev Med Pharmacol Sci 25:2748-2751. https://doi. org/10.26355/eurrev_202103_25438 
97. Wang EK, Chen CM, Hassan MM, Almogren A (2020) A deep learning based medical image segmentation technique in internet-of-medical-things domain. Futur Gener Comput Syst 108:135-144

98. Wang L, Lin ZQ, Wong A (2020) Covid-net: A tailored deep convolutional neural network design for detection of covid-19 cases from chest X-ray images. Sci Rep 10(1):1-12

99. Wang S, Kang B, Ma J, Zeng X, Xiao M, Guo J, Cai M, Yang J, Li Y, Meng X, Xu B (2021) A deep learning algorithm using CT images to screen for Corona virus disease (COVID-19). Eur Radiol 31:1-9

100. Xie W, Noble JA, Zisserman A (2018) Microscopy cell counting and detection with fully convolutional regression networks. Comput Methods Biomech Biomed Eng Imaging Vis 6(3):283-292

101. Xu X, Jiang X, Ma C, Du P, Li X, Lv S, ... Li L (2020) A deep learning system to screen novel coronavirus disease 2019 pneumonia. Engineering 6(10):1122-1129

102. Yao Q, Xiao L, Liu P, Zhou SK (2021) Label-free segmentation of COVID-19 lesions in lung CT. IEEE Trans Med Imaging 40:2808-2819

103. Ye C, Wang W, Zhang S, Wang K (2019) Multi-depth fusion network for whole-heart CT image segmentation. IEEE Access 7:23421-23429

104. Yellow fungus cases reported in UP: Know why it can prove more dangerous than black, white fungus (n.d.). [online]. Available: https://timesofindia.indiatimes.com/life-style/health-fitness/health-news/yellowfungus-cases-reported-in-up-know-why-it-can-prove-more-dangerous-than-black-fungus-white-fungus/ photostory/82905030.cms. Accessed 3 Mar 2021

105. Zhang K, Zhang L, Lam KM, Zhang D (2015) A level set approach to image segmentation with intensity inhomogeneity. IEEE Trans Cybern 46(2):546-557

106. Zhang J, Xie Y, Li Y, Shen C, Xia Y (2020) Covid-19 screening on chest X-ray images using deep learning based anomaly detection. arXiv preprint arXiv:2003.12338

107. Zheng C, Deng X, Fu Q, Zhou Q, Feng J, Ma H, ... Wang X (2020) Deep learning-based detection for COVID-19 from chest CT using weak label. MedRxiv

Publisher's note Springer Nature remains neutral with regard to jurisdictional claims in published maps and institutional affiliations. 